\title{
NONLINEAR BOUND STATES ON WEAKLY HOMOGENEOUS SPACES
}

\author{
HANS CHRISTIANSON, JEREMY MARZUOLA, JASON METCALFE, \\ AND MICHAEL TAYLOR
}

\begin{abstract}
We prove the existence of ground state solutions for a class of nonlinear elliptic equations, arising in the production of standing wave solutions to an associated family of nonlinear Schrödinger equations. We examine two constrained minimization problems, which give rise to such solutions. One yields what we call $F_{\lambda}$-minimizers, the other energy minimizers. We produce such ground state solutions on a class of Riemannian manifolds called weakly homogeneous spaces, and establish smoothness, positivity, and decay properties. We also identify classes of Riemannian manifolds with no such minimizers, and classes for which essential uniqueness of positive solutions to the associated elliptic PDE fails.
\end{abstract}

\section{INTRODUCTION}

Let $M$ be a Riemannian manifold of dimension $n \geqslant 2$ (possibly with boundary) with $\mathcal{C}^{\infty}$ bounded geometry. Eventually we will impose the additional assumption of weak homogeneity, but for now we work in the generality of bounded geometry. We consider the nonlinear Schrödinger equation on $M$, and in particular, we are interested in studying the existence of nonlinear bound states (standing waves).

The nonlinear Schrödinger equations we consider are given by

$$
\left\{\begin{array}{l}
i v_{t}+\Delta v+|v|^{p-1} v=0, x \in M \\
v(0, x)=v_{0}(x)
\end{array}\right.
$$

where $\Delta$ is the Laplace-Beltrami operator on $M$. If $\partial M \neq \emptyset$, we might impose the Dirichlet or Neumann boundary condition at $\partial M$. A nonlinear bound state is a choice of initial condition $u_{\lambda}(x)$ such that

$$
v(t, x)=e^{i \lambda t} u_{\lambda}(x)
$$

satisfies (1.0.1) with initial data $v(0, x)=u_{\lambda}(x)$. Such a solution is also called a ground state, a standing wave, or a solitary wave, or, sometimes, a soliton. Plugging in this ansatz yields the following elliptic equation for $u_{\lambda}$ :

$$
-\Delta u_{\lambda}+\lambda u_{\lambda}-\left|u_{\lambda}\right|^{p-1} u_{\lambda}=0 .
$$

Similarly, seeking a standing wave solution to a nonlinear Klein-Gordon equation,

$$
v_{t t}-\Delta v+\sigma^{2} v-|v|^{p-1} v=0, \quad v(t, x)=e^{i \mu t} u(x),
$$

leads to (1.0.3), with $\lambda=\sigma^{2}-\mu^{2}$.

In studying (1.0.3), we will assume

$$
\lambda>-\delta_{0},
$$


given that the spectrum of $-\Delta$ is contained in a semi-infinite interval

$$
\operatorname{Spec}(-\Delta) \subset\left[\delta_{0}, \infty\right)
$$

for some $\delta_{0} \geqslant 0$.

We will analyze two methods of establishing the existence of a solution to (1.0.3). One is to mimimize the functional

$$
F_{\lambda}(u)=\|\nabla u\|_{L^{2}}^{2}+\lambda\|u\|_{L^{2}}^{2}
$$

subject to the constraint

$$
J_{p}(u)=\int_{M}|u|^{p+1} d V=\beta,
$$

with $\beta \in(0, \infty)$ fixed. For this, we will require

$$
p \in\left(1,1+\frac{4}{n-2}\right), \quad \text { i.e., } p+1 \in\left(2, \frac{2 n}{n-2}\right) .
$$

The other is to minimize the energy

$$
E(u)=\frac{1}{2}\|\nabla u\|_{L^{2}}^{2}-\frac{1}{p+1} \int_{M}|u|^{p+1} d V,
$$

subject to the constraint on the "mass"

$$
Q(u)=\|u\|_{L^{2}}^{2}=\beta,
$$

with $\beta \in(0, \infty)$ fixed. For this, we require the more stringent condition

$$
p \in\left(1,1+\frac{4}{n}\right) .
$$

The energy functional (1.0.10) is conserved for sufficiently regular solutions to the nonlinear Schrödinger equation (1.0.1), which imparts special importance to energy minimizers.

We preview these approaches in more detail.

1.1. $F_{\lambda}$ minimizers. We make the hypotheses (1.0.5) and (1.0.9), and desire to minimize $F_{\lambda}(u)$ over $u \in H^{1}(M)$, subject to the constraint (1.0.8). We take $H^{1}(M)$ to be the $L^{2}$-Sobolev space of complex valued functions on $M$, however, with the structure of a vector space over $\mathbb{R}$. Here and below, if $\partial M \neq \emptyset$ and we impose the Dirichlet condition, we take $H^{1}(M)$ to mean $H_{0}^{1}(M)$. In certain cases, such as $\$ 2.3$. where the Dirichlet boundary condition is central, we use the notation $H_{0}^{1}(M)$, for emphasis.

If $u, v \in H^{1}(M)$, we have

$$
\begin{aligned}
& \left.\frac{d}{d \tau} F_{\lambda}(u+\tau v)\right|_{\tau=0}=2 \operatorname{Re}(-\Delta u+\lambda u, v), \\
& \left.\frac{d}{d \tau} J_{p}(u+\tau v)\right|_{\tau=0}=(p+1) \operatorname{Re} \int|u|^{p-1} u \bar{v} d V .
\end{aligned}
$$

If $u \in H^{1}(M)$ is a constrained minimizer, then

$$
\begin{aligned}
v & \in H^{1}(M) \text { and } \operatorname{Re} \int_{M}|u|^{p-1} u \bar{v} d V=0 \\
& \Longrightarrow \operatorname{Re}(-\Delta u+\lambda u, v)=0
\end{aligned}
$$


so the two elements of $H^{-1}(M),-\Delta u+\lambda u$ and $|u|^{p-1} u$, are linearly dependent over $\mathbb{R}$. Hence there exists a real constant $K_{0}$ such that

$$
-\Delta u+\lambda u=K_{0}|u|^{p-1} u,
$$

with equality holding in $H^{-1}(M)$. To determine $K_{0}$, we pair each side of this equation with $u$ and use $H^{1}-H^{-1}$ duality:

$$
\|\nabla u\|_{L^{2}}^{2}+\lambda\|u\|_{L^{2}}^{2}=K_{0} \int_{M}|u|^{p+1} d V=\beta K_{0} .
$$

Hence

$$
K_{0}=\frac{1}{\beta} \inf \left\{F_{\lambda}(u): u \in H^{1}(M), J_{p}(u)=\beta\right\} .
$$

Given the existence of such an infimizer, it follows that

$$
K_{0}>0 .
$$

Now if $u$ solves (1.1.3), then $u_{a}(x)=a u(x)$ solves

$$
-\Delta u_{a}+\lambda u_{a}=|a|^{-(p-1)} K_{0}\left|u_{a}\right|^{p-1} u_{a},
$$

so that we can solve

$$
-\Delta u+\lambda u=K|u|^{p-1} u
$$

for any $K>0$.

1.2. Energy minimizers. We make the hypothesis (1.0.12) on $p$ and desire to minimize $E(u)$, subject to the constraint (1.0.11). If $u, v \in H^{1}(M)$, we have

$$
\begin{aligned}
& \left.\frac{d}{d \tau} E(u+\tau v)\right|_{\tau=0}=\operatorname{Re}\left(-\Delta u-|u|^{p-1} u, v\right), \\
& \left.\frac{d}{d \tau} Q(u+\tau v)\right|_{\tau=0}=2 \operatorname{Re}(u, v) .
\end{aligned}
$$

If $u \in H^{1}(M)$ is a constrained minimizer, then

$$
\begin{aligned}
& v \in H^{1}(M), \quad \operatorname{Re}(u, v)=0 \\
& \quad \Longrightarrow \operatorname{Re}\left(\Delta u+|u|^{p-1} u, v\right)=0,
\end{aligned}
$$

and it follows that there exists $\lambda \in \mathbb{R}$ such that $\Delta u+|u|^{p-1} u=\lambda u$, or equivalently,

$$
-\Delta u+\lambda u=|u|^{p-1} u \text {. }
$$

1.3. Background. Before describing the structure of the main body of this paper, we recall some previous work on ground state solutions to (1.0.3), and describe how we plan to extend the scope. There is a large literature on such problems, when $M$ is Euclidean space $\mathbb{R}^{n}$ or a bounded domain in $\mathbb{R}^{n}$, addressing questions of existence, uniqueness, and stability. We mention a few of these, referring to them for further references.

Pohozaev Poh65] studied the case where $M$ is a bounded domain in $\mathbb{R}^{n}$, for $p$ as in (1.0.9) (also allowing for more general nonlinearities), producing $F_{\lambda}$-minimizers. These results extend readily to general compact Riemannian manifolds with boundary. Strauss [Str77] obtained solutions on $\mathbb{R}^{n}$, essentially via $F_{\lambda}$-minimizers. This work was followed by [BL83]. This paper constructs a minimizer of $\|\nabla u\|_{L^{2}}^{2}$, subject to the constraint

$$
\int_{\mathbb{R}^{n}} G(u(x)) d x=1
$$


where

$$
G(u)=\int_{0}^{u} g(t) d t, \quad g(u)=|u|^{p-1} u-\lambda u .
$$

Both Str77 and BL83] considered more general nonlinearities. A key device in both papers was a symmetrization technique: if $u \in H^{1}\left(\mathbb{R}^{n}\right)$, then replacing $u$ by its decreasing radial rearrangement $u^{*}$ leaves $\|u\|_{L^{2}}$ and $\|u\|_{L^{p+1}}$ unchanged and does not increase $\|\nabla u\|_{L^{2}}$. In Str77] this was demonstrated directly. As noted in [BL83, it also follows from BLL74, via an argument using heat kernel monotonicity and a rearrangement inequality. From this result, it suffices to seek a minimizer within the class of radial functions in $H^{1}\left(\mathbb{R}^{n}\right)$, where estimates implying compactness are available. The papers Lio84a - Lio84b introduced the concentration-compactness method and applied it to a number of problems, including a construction of $F_{\lambda^{-}}$ minimizers, and also a discussion of energy minimizers.

Another approach was taken in Wei83. There a solution to (1.0.3) was constructed to maximize the Weinstein functional

$$
W(u)=\frac{\|u\|_{L^{p+1}}^{p+1}}{\|u\|_{L^{2}}^{\alpha}\|\nabla u\|_{L^{2}}^{\beta}},
$$

over $u \in H^{1}\left(\mathbb{R}^{n}\right)$, under the hypothesis (1.0.9), where

$$
\alpha=2-\frac{(n-2)(p-1)}{2}, \beta=\frac{n(p-1)}{2},
$$

(Note that $\alpha, \beta>0$ and $\alpha+\beta=p+1$.) The supremum $W_{\max }$ is the best constant in the Gagliardo-Nirenberg inequality

$$
\|u\|_{L^{p+1}}^{p+1} \leq W_{\max }\|u\|_{L^{2}}^{\alpha}\|\nabla u\|_{L^{2}}^{\beta}, \quad u \in H^{1}\left(\mathbb{R}^{n}\right) .
$$

See also Appendix B of [Tao06] for a presentation of this work, and 4.3 of this paper for another demonstration of the existence of a maximizer.

There are infinitely many solutions to (1.0.3) on $\mathbb{R}^{n}$, but the ground state is (up to a constant factor) positive everywhere, and there are results on uniqueness (up to isometries) of positive solutions, culminating in Kwo89, McL93, and CJ93. Work on orbital stability of solutions to the nonlinear Schrödinger equation (1.0.1) with initial data given by such ground states includes [CL82, Wei85, Wei86, and GSS90. The hypothesis that the ground state be energy minimizing plays a major role in these results, and this motivates our interest in establishing existence of energy minimizers as well as $F_{\lambda}$-minimizers. (Stability issues for $F_{\lambda}$-minimizers that are not energy minimizers are different; cf. [Sch09].)

Moving beyond the cases of bounded domains and Euclidean space, the case where $M$ is hyperbolic space $\mathcal{H}^{n}$, a symmetric space of constant negative sectional curvature, was treated independently in [MS08] and by two of us, in CM10. The techniques in these two papers are rather different. Following BL83, the paper CM10 finds a minimizer of $\|\nabla u\|_{L^{2}}^{2}$, subject to the constraint (1.3.1). It uses a mixture of a symmetrization technique and a concentration-compactness argument. In this case, the fact that $u \mapsto u^{*}$ does not increase $\|\nabla u\|_{L^{2}}$ was established in CM10, via heat kernel monotonicity in the setting of $\mathcal{H}^{n}$ plus the extension of the rearrangement inequality of BLL74, to the setting of hyperbolic space, given in Bec92] (following the extension to the sphere given in BT76]). The paper MS08 tackles existence via a minimization process essentially equivalent to finding an $F_{\lambda^{-}}$ minimizer, and makes use of the Ekeland principle. Their paper also has existence 
results for the case when $-\lambda$ is at the bottom of the spectrum of $-\Delta$, and also results on both existence and non-existence for critical $p=(n+2) /(n-2)$. Furthermore, MS08 establishes uniqueness of positive solutions (up to isometries) in this setting.

In this paper, we work on the following class of complete Riemannian manifolds $M$, possibly with boundary. We assume there is a group $G$ of isometries of $M$ and a number $D>0$ such that for every $x, y \in M$, there exists $g \in G$ such that $\operatorname{dist}(x, g(y)) \leq D$. We call such a manifold $M$ a weakly homogeneous space. We give some examples of such spaces.

First, if $M$ is compact (perhaps with boundary), such a condition holds, even if only the identity map on $M$ is an isometry. Next, if $M$ is a homogeneous space, that is, $M$ has a transitive group of isometries (such as $\mathbb{R}^{n}$ or $\mathcal{H}^{n}$, among many other richly studied examples), such a condition holds. We mention some examples that are neither compact nor homogeneous.

Example 1. Pick $R \in(0,1 / 2)$ and take

$$
M=\mathbb{R}^{n} \backslash \bigcup_{k \in \mathbb{Z}^{n}} B_{R}(k) .
$$

EXAmple 2. Let $M^{b}$ be a compact Riemannian manifold (perhaps with boundary), and let $M$ be the universal covering space of $M^{b}$, with the induced metric tensor. More generally, $M$ can be any covering space of $M^{b}$. This class of examples includes Example 1 as a special case.

1.4. Plan of the paper. In $\oiint 2$ we prove existence of a minimizer $u \in H^{1}(M)$ to $F_{\lambda}(u)$, subject to the constraint (1.0.8), given $p$ as in (1.0.9), and establish some useful properties of such solutions, when $M$ is a weakly homogeneous space. The first use of the constraint on $p$ is to get

$$
I_{\beta}=\inf \left\{F_{\lambda}(u): u \in H^{1}(M), J_{p}(u)=\beta\right\}>0 .
$$

We then apply the concentration-compactness method of P.-L. Lions, suitably extended to the manifold setting. Concentration is established in 2.1 and from there compactness and existence of $F_{\lambda}$-minimizers is proven in $\$ 2.2$, The concentration argument works whenever $M$ has bounded geometry. It is in passing to the compactness argument that we use the weak homogeneity. Regarding the necessity of some geometrical constraint, we give examples in $\$ 2.3$ of Riemannian manifolds (with boundary) with bounded geometry, for which there is not such an $F_{\lambda}$-minimizer.

Section 2.4 establishes smoothness of minimizers, and $\$ 2.5$ is devoted to showing that every real valued minimizer $u$ is either $>0$ on $M$ or $<0$ on $M$, and obtaining some Harnack-type estimates. Further global bounds on positive minimizers are obtained in $\$ 2.6$. In $\S 2.42 .6$ we assume for simplicity that $\partial M=\emptyset$. Some local regularity estimates for nonempty boundary are given in Tay11, in the setting of Lipschitz domains.

In 93 we prove existence of a minimizer $u \in H^{1}(M)$ to the energy $E(u)$, subject to the constraint (1.0.11), given $p$ as in (1.0.12), when $M$ is a weakly homogeneous space, under one further condition. Namely, we require

$$
\mathcal{I}_{\beta}=\inf \left\{E(u): u \in H^{1}(M), Q(u)=\beta\right\}
$$


to satisfy

$$
\mathcal{I}_{\beta}<0 .
$$

(Replace $H^{1}(M)$ by $H_{0}^{1}(M)$ when using the Dirichlet boundary condition.) Again we use a concentration-compactness argument. In 3.1 we show that (1.4.3) forces concentration, and we discuss existence of energy minimizers for this in $\$ 3.2$. We mention that energy minimizers are (constant multiples of) positive functions on $M$, and many of the estimates of $\$ \$ 2.42 .6$ apply here, as seen in $\$ 3.4$, following a discussion of manifolds with no energy minimizers in 3.3 . We give a formula for the second variation of energy, for energy minimizers, in 33.5 and for $F_{\lambda}$-minimizers in $\$ 3.6$ and apply these formulas in $\$ 3.7$ to results on operators $L_{ \pm}$. These results in turn are applied in 3.8 to some results on orbital stability.

In $\$$ we take another look at the symmetrization method, mentioned above in the context of $\mathbb{R}^{n}$. After a discussion of the rearrangement lemma in 4.1 in 4.2 we pursue a uniform approach to a proof of existence of $F_{\lambda}$-minimizers, valid for $M=\mathcal{H}^{n}$ and for $\mathbb{R}^{n}$. This proof is adapted from CM10, but it incorporates simplifications that allow us to avoid completely the concentration-compactness argument in this context. In 4.3 we apply the symmetrization approach to maximizing $W(u)$ in (1.3.3). In 4.4 we discuss such a symmetrization approach to the existence of energy minimizers, when $M=\mathcal{H}^{n}$ or $\mathbb{R}^{n}(n \geq 2)$. We see that this approach succeeds up to a point, but there appears a gap, and it remains to be seen whether the concentration-compactness argument can be avoided in this setting.

We have four appendices, containing supporting material related to the results

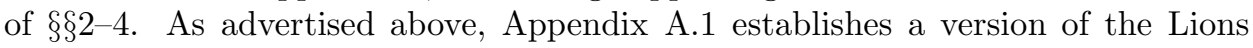
concentration-compactness argument. We work in a general class of measured metric spaces, which in particular includes the Riemannian manifolds with bounded geometry arising in $\S ₫ 2$, 3

In 3 A.2 we look at the behavior of the energy of positive solutions to (1.0.3) on Euclidean space $\mathbb{R}^{n}$. We show that if $1<p<1+4 / n$ then all such solutions (with $\lambda>0$ ) have negative energy and are energy minimizing within their mass class, while if $1+4 / n<p<(n+2) /(n-2)$, there can be no energy minimizer within its mass class, at any positive mass. In A.3. we discuss conditions under which $F_{\lambda}$-minimizers can be shown to have positive energy $(E(u)>0)$, even when $1<p<1+4 / n$. In particular, we show that all such minimizers on hyperbolic space $\mathcal{H}^{n}$ associated to $\lambda \leq 0$ have positive energy.

In $\$$ A.4 we examine some positive solutions to (1.0.3) that are not $F_{\lambda}$-minimizers, and exhibit some cases of essential non-uniqueness of such positive solutions.

\section{2. $F_{\lambda}$ MINIMIZERS}

We take up the task of minimizing $F_{\lambda}(u)$, subject to the constraint $J_{p}(u)=\beta$. Observe that (1.0.5)-(1.0.6) imply

$$
F_{\lambda}(u) \simeq\|u\|_{H^{1}(M)}^{2} .
$$

The hypothesis (1.0.9) on the range of $p$ implies

$$
H^{1}(M) \subset L^{p+1}(M) .
$$

Hence there exists a constant $C>0$ such that

$$
\|u\|_{L^{p+1}}^{2} \leqslant C F_{\lambda}(u),
$$


so that

$$
I_{\beta}=\inf \left\{F_{\lambda}(u): u \in H^{1}(M), J_{p}(u)=\beta\right\}>0 .
$$

(Recall from $\$ 1$ that $H^{1}(M)$ stands for $H_{0}^{1}(M)$ if $\partial M \neq \emptyset$ and we use the Dirichlet boundary condition.)

Suppose $\left\{u_{\nu}\right\} \subset H^{1}(M)$ is a sequence satisfying

$$
J_{p}\left(u_{\nu}\right)=\beta, \quad F_{\lambda}\left(u_{\nu}\right) \leqslant I_{\beta}+\frac{1}{\nu} .
$$

Passing to a subsequence if necessary, we have

$$
u_{\nu} \rightarrow u \in H^{1}(M)
$$

converging in the weak topology. Rellich's theorem implies

$$
H^{1}(M) \hookrightarrow L^{p+1}(\Omega)
$$

is compact provided $\Omega$ is relatively compact. This implies that, for such $\Omega$,

$$
u_{\nu} \rightarrow u \text { in the } L^{p+1}(\Omega) \text { norm. }
$$

If our manifold $M$ is compact, we can take $M=\Omega$ and the minimization problem is simple. We are interested in the non-compact setting, for which further argument is necessary.

To carry this out, we use the concentration-compactness method of Lions Lio84a, Lio84b. We set up the concentration-compactness argument in $\$ 2.1$ and establish concentration. In 22.2 we establish compactness and prove existence of $F_{\lambda^{-}}$ minimizers, when $M$ is a weakly homogeneous space. In $\$ 2.3$ we exhibit some manifolds with bounded geometry on which there are no $F_{\lambda}$-minimizers. In $\$ ₫ 2.4$ 2.6 we study smoothness, positivity, and decay estimates on the solutions; in these last three sections we assume for simplicity that $\partial M=\emptyset$.

2.1. Concentration. In this section we again assume only that $M$ is a smooth manifold with $\mathcal{C}^{\infty}$ bounded geometry. The enemy of finding a minimizer is the possibility of minimizing sequences escaping to spatial infinity. We will show that some minimizing subsequence concentrates, which we will define shortly.

Let us first record a basic Lemma of concentration compactness. This is given in Lions Lio84a, pp. 115-117, in the context of Euclidean space. Here we state the result in the context of a Riemannian manifold. In Appendix A.1 we establish the result in an even more general setting.

Lemma 2.1.1. Let $M$ be a Riemannian manifold. Fix $\beta \in(0, \infty)$. Let $\left\{u_{\nu}\right\} \in$ $L^{p+1}(M)$ be a sequence satisfying $\int\left|u_{\nu}\right|^{p+1} d V=\beta$. Then, after extracting a subsequence, one of the following three cases holds:

(i) Vanishing. If $B_{R}(y)=\{x \in M$ : $\operatorname{dist}(x, y) \leqslant R\}$ is the closed ball, then for all $0<R<\infty$,

$$
\lim _{\nu \rightarrow \infty} \sup _{y \in M} \int_{B_{R}(y)}\left|u_{\nu}\right|^{p+1} d V=0 .
$$

(ii) Concentration. There exists a sequence of points $\left\{y_{\nu}\right\} \subset M$ with the property that for each $\epsilon>0$, there exists $R(\epsilon)<\infty$ such that

$$
\int_{B_{R(\epsilon)}\left(y_{\nu}\right)}\left|u_{\nu}\right|^{p+1} d V>\beta-\epsilon .
$$


(iii) Splitting. There exists $\alpha \in(0, \beta)$ with the following properties: For each $\epsilon>0$, there exists $\nu_{0} \geqslant 1$ and sets $E_{\nu}^{\sharp}, E_{\nu}^{b} \subset M$ such that

$$
\operatorname{dist}\left(E_{\nu}^{\sharp}, E_{\nu}^{b}\right) \rightarrow \infty \text { as } \nu \rightarrow \infty,
$$

and

$$
\left.\left|\int_{E_{\nu}^{\sharp}}\right| u_{\nu}\right|^{p+1} d V-\alpha|<\epsilon,| \int_{E_{\nu}^{b}}\left|u_{\nu}\right|^{p+1} d V-(\beta-\alpha) \mid<\epsilon .
$$

We now show that for a minimizing sequence in our problem, the vanishing phenomenon cannot occur. The proof uses the following lemma, essentially given in Lio84b].

Lemma 2.1.2. Assume $\left\{u_{\nu}\right\}$ is bounded in $H^{1}(M)$, and

$$
\lim _{\nu \rightarrow \infty} \sup _{y \in M} \int_{B_{R}(y)}\left|u_{\nu}\right|^{2} d V=0 \text {, for some } R>0 .
$$

Then

$$
2<r<\frac{2 n}{n-2} \Longrightarrow\left\|u_{\nu}\right\|_{L^{r}(M)} \rightarrow 0
$$

Proof. This is a special case of Lemma I.1 on p. 231 of [Lio84b]. Actually, the lemma there is established for $M=\mathbb{R}^{n}$. However, the only two geometric properties used in the proof in Lio84b are the existence of Sobolev embeddings on balls of radius $R>0$, and the fact that there exists $m<\infty$ such that $\mathbb{R}^{n}$ has a covering by balls of radius $R$ in such a way that each point is contained in at most $m$ balls. These two facts hold on every smooth Riemannian manifold with $\mathcal{C}^{\infty}$ bounded geometry.

Proposition 2.1.3. Suppose $\left\{u_{\nu}\right\}$ is a minimizing sequence. Then no subsequence can satisfy 2.1.1).

Proof. If (2.1.1) holds, then so does (2.1.5), by Hölder's inequality on finite measure balls. Then (2.1.6) holds with $r=p+1$ (recall (1.0.9)). This contradicts the assumption that $J_{p}(u)=\beta>0$.

To show that splitting is impossible, we start by showing that $I_{\beta}$, given by (1.4.1), has the property that, for all $\beta>0$,

$$
I_{\beta}<I_{\eta}+I_{\beta-\eta} \text {, for any } \eta \in(0, \beta) \text {. }
$$

Lions gives (2.1.7) a key role in results of [Lio84a - Lio84b, showing that, in various situations, splitting cannot occur. In fact, in this case we have much more structure.

Proposition 2.1.4. For all $\beta>0$, we have

$$
I_{\beta}=I_{1} \beta^{2 /(p+1)} .
$$

Proof. Suppose $u_{\nu}$ satisfies (2.0.5), so

$$
J_{p}\left(u_{\nu}\right)=\beta, \quad F_{\lambda}\left(u_{\nu}\right) \rightarrow I_{\beta} .
$$

Then for $a>0$,

$$
J_{p}\left(a u_{\nu}\right)=a^{p+1} \beta, \quad F_{\lambda}\left(a u_{\nu}\right) \rightarrow a^{2} I_{\beta} .
$$


Hence

$$
\gamma=a^{p+1} \beta \Longrightarrow I_{\gamma} \leqslant a^{2} I_{\beta} .
$$

Now if we replace $\beta$ by $\gamma$ and $a$ by $a^{-1}$, we get $I_{\beta} \leqslant a^{-2} I_{\gamma}$, i.e., $I_{\gamma} \geqslant a^{2} I_{\beta}$, which together with (2.1.11) implies

$$
\gamma=a^{p+1} \beta \Longrightarrow I_{\gamma}=a^{2} I_{\beta},
$$

and proves the proposition.

Note that since $C F_{\lambda}(u) \geqslant \int|u|^{p+1} d V$, we have $I_{1}>0$, so $I_{\beta}>0$ for every $\beta>0$, and (2.1.7) follows.

Remark. The proof of Proposition 2.1.4 implies that $F_{\lambda}$-minimizers scale to other $F_{\lambda}$-minimizers. We state this formally.

Corollary 2.1.5. If $\beta>0$ and $u$ minimizes $F_{\lambda}$, subject to the constraint $J_{p}(u)=\beta$, and if $\kappa>0$, then $u_{\kappa}=\kappa u$ minimizes $F_{\lambda}$, subject to the constraint $J_{p}\left(u_{\kappa}\right)=\kappa^{p+1} \beta$.

Let us now show that splitting cannot occur for a minimizing subsequence. Suppose on the contrary that there exists $\alpha \in(0, \beta)$ and for each $\epsilon>0$, sets $E_{\nu}^{\sharp}, E_{\nu}^{b} \subset M$ such that (2.1.3)-(2.1.4) occur. Choose $\epsilon>0$ sufficiently small that

$$
I_{\beta}<I_{\alpha}+I_{\beta-\alpha}-C_{1} \epsilon,
$$

where $C_{1}>0$ is a sufficiently large constant to be fixed later. Since $\left\|u_{\nu}\right\|_{H^{1}(M)}$ is uniformly bounded, if follows from (2.1.3) that there exists $\nu_{1}$ such that $\nu \geqslant \nu_{1}$ implies

$$
\int_{S_{\nu}}\left|u_{\nu}\right|^{2} d V<\epsilon
$$

where $S_{\nu}$ is a set of the form

(2.1.15) $S_{\nu}=\left\{x \in M: d_{\nu}<\operatorname{dist}\left(x, E_{\nu}^{\sharp}\right) \leqslant d_{\nu}+2\right\} \subset M \backslash\left(E_{\nu}^{\sharp} \cup E_{\nu}^{b}\right)$,

for some $d_{\nu}>0$. For $r>0$ and $\nu \geqslant \nu_{1}$, set

$$
\widetilde{E}_{\nu}(r)=\left\{x \in M: \operatorname{dist}\left(x, E_{\nu}^{\sharp}\right) \leqslant r\right\},
$$

so that $S_{\nu}=\widetilde{E}_{\nu}\left(d_{\nu}+2\right) \backslash \widetilde{E}\left(d_{\nu}\right)$. Define functions $\chi_{\nu}^{\sharp}$ and $\chi_{\nu}^{b}$ by

$$
\chi_{\nu}^{\sharp}(x)=\left\{\begin{array}{l}
1, \quad \text { if } x \in \widetilde{E}_{\nu}\left(d_{\nu}\right), \\
1-\operatorname{dist}\left(x, \widetilde{E}_{\nu}\left(d_{\nu}\right)\right), \quad \text { if } x \in \widetilde{E}_{\nu}\left(d_{\nu}+1\right), \\
0, \quad \text { if } x \notin \widetilde{E}_{\nu}\left(d_{\nu}+1\right),
\end{array}\right.
$$

and

$$
\chi_{\nu}^{b}(x)=\left\{\begin{array}{l}
0, \quad \text { if } x \in \widetilde{E}_{\nu}\left(d_{\nu}+1\right), \\
\operatorname{dist}\left(x, \widetilde{E}_{\nu}\left(d_{\nu}\right)\right), \quad \text { if } x \in \widetilde{E}_{\nu}\left(d_{\nu}+2\right), \\
1, \quad \text { if } x \notin \widetilde{E}_{\nu}\left(d_{\nu}+2\right),
\end{array}\right.
$$

These functions are both Lipschitz with Lipschitz constant 1 and almost disjoint supports. Set $u_{\nu}^{\sharp}=\chi_{\nu}^{\sharp} u_{\nu}$ and $u_{\nu}^{b}=\chi_{\nu}^{b} u_{\nu}$. Since $0 \leqslant \chi_{\nu}^{\sharp}+\chi_{\nu}^{b} \leqslant 1$, we have

$$
J_{p}\left(u_{\nu}^{\sharp}\right)+J_{p}\left(u_{\nu}^{b}\right) \leqslant \int\left(\chi_{\nu}^{\sharp}+\chi_{\nu}^{b}\right)\left|u_{\nu}\right|^{p+1} d V \leqslant J_{p}\left(u_{\nu}\right)=\beta .
$$

Also, of course if $\lambda \geqslant 0$,

$$
\lambda\left\|u_{\nu}^{\sharp}\right\|_{L^{2}}^{2}+\lambda\left\|u_{\nu}^{b}\right\|_{L^{2}}^{2} \leqslant \lambda\left\|u_{\nu}\right\|_{L^{2}}^{2},
$$


while if $\lambda<0$, we have

$$
|\lambda|\left(\left\|u_{\nu}\right\|_{L^{2}}^{2}-\left\|u_{\nu}^{\sharp}\right\|_{L^{2}}^{2}-\left\|u_{\nu}^{b}\right\|_{L^{2}}^{2}\right)<|\lambda| \epsilon .
$$

We have $\nabla u_{\nu}^{\sharp}=\chi_{\nu}^{\sharp} \nabla u_{\nu}+\left(\nabla \chi_{\nu}^{\sharp}\right) u_{\nu}$ and similarly for $u_{\nu}^{b}$, and $\left|\nabla \chi_{\nu}^{\sharp}\right| \leqslant 1$ except for a set of measure zero, so

$$
\left\|\nabla u_{\nu}^{\sharp}\right\|_{L^{2}}^{2}+\left\|\nabla u_{\nu}^{b}\right\|_{L^{2}}^{2} \leqslant\left\|\nabla u_{\nu}\right\|_{L^{2}}^{2}+\int_{S_{\nu}}\left|u_{\nu}\right|^{2} d V .
$$

As a consequence, we have

$$
F_{\lambda}\left(u_{\nu}^{\sharp}\right)+F_{\lambda}\left(u_{\nu}^{b}\right) \leqslant F_{\lambda}\left(u_{\nu}\right)+\epsilon+\sigma(\lambda) \epsilon,
$$

where

$$
\sigma(\lambda)=\left\{\begin{array}{l}
|\lambda|, \quad-\delta_{0}<\lambda<0 \\
0, \quad \lambda \geqslant 0 .
\end{array}\right.
$$

Using the support properties of $u_{\nu}^{\sharp}, u_{\nu}^{b}$ together with (2.1.3)-(2.1.4) yields

$$
\left|J_{p}\left(u_{\nu}^{\sharp}\right)-\alpha\right|, \quad\left|J_{p}\left(u_{\nu}^{b}\right)-(\beta-\alpha)\right| \leqslant 3 \epsilon .
$$

Combining (2.1.23)-(2.1.25), and letting $\nu \rightarrow \infty$, we get

$$
I_{\alpha}+I_{\beta-\alpha} \leqslant I_{\beta}+C_{2} \epsilon,
$$

where $C_{2}$ depends only on $\delta_{0}>0$, the bottom of the spectrum of $-\Delta$. Hence if $C_{1}>C_{2}$ is chosen sufficiently large in (2.1.13) (which simply amounts to producing $\epsilon$ sufficiently small), we contradict (2.1.13). This, together with Proposition 2.1.3 yields the following proposition, which states that for a minimizing sequence $u_{\nu}$, only the concentration phenomenon can occur.

Proposition 2.1.6. Let $\left\{u_{\nu}\right\}$ be a minimizing sequence of $I_{\lambda}$. Then every subsequence of the $\left\{u_{\nu}\right\}$ has a further subsequence (which we will continue to denote by $\left.\left\{u_{\nu}\right\}\right)$ with the following property. There exists a sequence $\left\{y_{\nu}\right\} \subset M$ and a function $\tilde{R}(\epsilon)$ such that for all $\nu$,

$$
\int_{B_{\tilde{R}(\epsilon)}\left(y_{\nu}\right)}\left|u_{\nu}\right|^{p+1} d V>\beta-\epsilon, \quad \forall \epsilon>0,
$$

Remark 2.1.7. It is very important to observe the following facts in Proposition 2.1.6. The sequence of points $\left\{y_{\nu}\right\}$ is independent of $\epsilon>0$, and the function $\tilde{R}(\epsilon)$ is independent of the index $\nu$.

Proposition [2.1.6 is about concentration along subsequences of a minimizing sequence. It holds on any $\mathcal{C}^{\infty}$ manifold $M$ with bounded geometry. In order to show that a minimizer actually exists, we need compactness, which will follow once we assume the additional structure of weak homogeneity on $M$. This will allow us to map the sequence $\left\{y_{\nu}\right\}$ into a compact region so that any subsequence which concentrates as in Proposition 2.1.6 will enjoy compact Sobolev embeddings by Rellich's theorem. 
2.2. Compactness and existence of minimizers. For this section, let $M$ be a smooth Riemannian manifold of dimension $n \geqslant 2$, satisfying the following weak homogeneity condition:

Definition 2.2.1. Let $M$ be a smooth, complete Riemannian manifold, possibly with boundary. We say $M$ is weakly homogeneous if there exists a group $G$ of isometries of $M$ and a number $D>0$ such that for every $x, y \in M$, there exists an element $g \in G$ such that $\operatorname{dist}(x, g(y)) \leqslant D$.

Remark. Such Riemannian manifolds necessarily have $\mathcal{C}^{\infty}$ bounded geometry.

We retain the hypotheses (1.0.6)-(1.0.9). Let $\left\{u_{\nu}\right\} \subset H^{1}(M)$ be a minimizing sequence for (2.0.4), that is,

$$
J_{p}\left(u_{\nu}\right)=\beta, \quad F_{\lambda}\left(u_{\nu}\right) \leqslant I_{\beta}+\frac{1}{\nu},
$$

where $F_{\lambda}$ is given by (1.0.7) and $J_{p}$ is given by (1.0.8) as usual. After passing to a subsequence if necessary, Proposition 2.1.6 shows we have points $\left\{y_{\nu}\right\} \subset M$ and a function $\tilde{R}(\epsilon)$ such that (2.1.27) holds.

We now fix a base point, or "origin" $o \in M$ and apply the weak homogeneity hypothesis: for each $\nu$, there exists $g_{\nu} \in G$ and $x_{\nu} \in B_{D}(o)$ such that $x_{\nu}=g_{\nu}\left(y_{\nu}\right)$. Set $v_{\nu}(x)=u_{\nu}\left(g_{\nu}^{-1}(x)\right)$, so that $v_{\nu}$ is now concentrated near $o$ instead of near $y_{\nu}$. The sequence $\left\{v_{\nu}\right\}$ satisfies

$$
J_{p}\left(v_{\nu}\right)=J_{p}\left(u_{\nu}\right)=\beta, \quad F_{\lambda}\left(v_{\nu}\right)=F_{\lambda}\left(u_{\nu}\right) \leqslant I_{\beta}+\frac{1}{\nu},
$$

and

$$
\int_{B_{\tilde{R}(\epsilon)+D}(o)}\left|v_{\nu}\right|^{p+1} d V>\beta-\epsilon, \quad \forall \epsilon>0 .
$$

Passing to a further subsequence, which we continue to denote by $\left\{v_{\nu}\right\}$, we have

$$
v_{\nu} \rightarrow v \text { weakly in } H^{1}(M) .
$$

Since $F_{\lambda}$ is comparable to the $H^{1}(M)$ norm squared, we have

$$
F_{\lambda}(v) \leqslant \liminf _{\nu \rightarrow \infty} F\left(v_{\nu}\right)=I_{\beta} .
$$

Similarly

$$
J_{p}(v) \leqslant \liminf _{\nu \rightarrow \infty} J_{p}\left(v_{\nu}\right)=\beta .
$$

On the other hand, by (2.0.7), we have for each $\epsilon>0$,

$$
v_{\nu} \rightarrow v, \text { in the } L^{p+1}\left(B_{\tilde{R}(\epsilon)+D}(o)\right) \text { norm, }
$$

so that (2.2.3) implies $J_{p}(v) \geqslant \beta$. Hence $J_{p}(v)=\beta$, which in turn implies

$$
F_{\lambda}(v)=I_{\beta}
$$

Let us summarize this argument in the following Proposition.

Proposition 2.2.2. If $M$ is a weakly homogeneous Riemannian manifold, and if (1.0.6) -(1.0.9) hold, then there exists a minimizer $v \in H^{1}(M)$ of $F_{\lambda}(v)$, subject to the constraint $J_{p}(v)=\beta$.

Remark. It follows from (2.2.8) that convergence $v_{\nu} \rightarrow v$ in (2.2.4 holds in norm in $H^{1}(M)$, hence in norm in $L^{p+1}(M)$. 
2.3. Manifolds with no $F_{\lambda}$ minimizers. We take

$$
\lambda>0, \quad \beta>0, \quad p \in\left(1,1+\frac{4}{n-2}\right),
$$

and

$$
M=\mathbb{R}^{n} \backslash K,
$$

where $K \subset \mathbb{R}^{n}$ is a smoothly bounded, compact set. We impose the Dirichlet boundary condition on $\partial M$, pick $\beta>0$, and seek minimizers of $F_{\lambda}(u)$, given

$$
u \in H_{0}^{1}(M), \quad J_{p}(u)=\beta .
$$

We will show that no such minimizer exists. To see this, first compare

$$
I_{\beta}(M)=\inf \left\{F_{\lambda}(u): u \in H_{0}^{1}(M), J_{p}(u)=\beta\right\}
$$

with

$$
I_{\beta}\left(\mathbb{R}^{n}\right)=\inf \left\{F_{\lambda}(u): u \in H^{1}\left(\mathbb{R}^{n}\right), J_{p}(u)=\beta\right\} .
$$

It is clear that $I_{\beta}(M) \geq I_{\beta}\left(\mathbb{R}^{n}\right)$, since (2.3.4) is an inf over a smaller set of functions.

On the other hand, one can take an $F_{\lambda}$-minimizer for $\mathbb{R}^{n}$, whose existence is classical (and follows as a special case from $\$ 2.2$ ), or, without appealing to this existence result, simply take an element $\tilde{u}_{\epsilon} \in H^{1}\left(\mathbb{R}^{n}\right)$ such that $J_{p}\left(\tilde{u}_{\epsilon}\right)=\beta$ and $F_{\lambda}\left(\tilde{u}_{\epsilon}\right) \geqslant I_{\beta}\left(\mathbb{R}^{n}\right)-\epsilon / 2$. Translate this function to be concentrated far away from $K$, and apply a cutoff to get an element $u_{\epsilon} \in H_{0}^{1}(M)$ such that $J_{p}\left(u_{\epsilon}\right)=\beta$ and $F_{\lambda}\left(u_{\epsilon}\right) \geqslant I_{\beta}\left(\mathbb{R}^{n}\right)-\epsilon$. Thus

$$
I_{\beta}(M)=I_{\beta}\left(\mathbb{R}^{n}\right) .
$$

We now prove the following.

Proposition 2.3.1. There does not exist $u \in H_{0}^{1}(M)$ such that $J_{p}(u)=\beta$ and $F_{\lambda}(u)=I_{\beta}(M)$.

Proof. Suppose such $u \in H_{0}^{1}(M)$ does exist. We can arrange that $u \geq 0$ on $M$. Set $v=u$ on $M, v=0$ on $K$. Then

$$
v \in H^{1}\left(\mathbb{R}^{n}\right), \quad J_{p}(v)=\beta, \quad F_{\lambda}(v)=I_{\beta}(M)=I_{\beta}\left(\mathbb{R}^{n}\right),
$$

so $v$ must be an $F_{\lambda}$-minimizer on $\mathbb{R}^{n}$. It is well known in the case of $\mathbb{R}^{n}$ (cf. 2.4 for more general results) that such $v$ must be $>0$ everywhere on $\mathbb{R}^{n}$, which presents a contradiction.

Remark. The $F_{\lambda}$-minimizing sequences described above exhibit concentration, consistent with results of $\$ 2.1$. The lack of an adequate family of isometries of $M$ in this setting prevents this from yielding a compactness result, and hence an $F_{\lambda}$-minimizer.

Remark. One readily obtains similar non-existence results for the complement of a compact set in a general noncompact, connected, weakly homogeneous space.

Remark. In Appendix A.4 we will build on the examples here to give examples of positive solutions to (1.0.3) that are not $F_{\lambda}$-minimizers, and examples of compact manifolds (with boundary) for which (1.0.3) has two geometrically inequivalent positive solutions. 
2.4. Smoothness of minimizers. As stated above, in $\S ₫ 2.42 .6$ we assume for simplicity that $\partial M=\emptyset$. We begin with a local regularity result. Let $\Omega \subset M$ be an open set, and assume $u \in H_{\mathrm{loc}}^{1}(\Omega)$ solves

$$
-\Delta u+\lambda u=f(u), \quad f(u)=K|u|^{p-1} u,
$$

with $p$ as in (1.0.9).

Proposition 2.4.1. Every solution $u \in H_{l o c}^{1}(\Omega)$ to (2.4.1) satisfies

$$
u \in C^{p+2}(\Omega)
$$

if $p \notin \mathbb{N}$. If $p$ is an odd integer, then $u \in C^{\infty}(\Omega)$. If $p$ is an even integer, $u \in C^{s}(\Omega)$ for all $s<p+2$, and if $p$ is an even integer and $u \geqslant 0$ on $\Omega$ then $u \in C^{\infty}(\Omega)$. Finally, for any $p$ satisfying (1.0.9), if $u$ is nowhere vanishing on $\Omega$, then $u \in C^{\infty}(\Omega)$.

Proof. This is a standard result, but we sketch the proof here in preparation for the global results in the sequel. Recall that for linear equations, we have elliptic regularity: for $1<q<\infty$ and $s \geqslant 0$,

$$
\begin{aligned}
-\Delta u+\lambda u=f \in H_{\mathrm{loc}}^{s, q}(\Omega) & \Longrightarrow u \in H_{\mathrm{loc}}^{s+2, q}(\Omega), \\
-\Delta u+\lambda u=f \in C^{s}(\Omega) & \left.\Longrightarrow u \in C^{s+2}(\Omega) \text { (if } s \notin \mathbb{Z}\right) .
\end{aligned}
$$

We also have Sobolev embeddings, such as

$$
\begin{aligned}
& H_{\mathrm{loc}}^{s, q}(\Omega) \subset L_{\mathrm{loc}}^{n q /(n-s q)}(\Omega), \quad 0<s<\frac{n}{q}, \\
& H_{\mathrm{loc}}^{s+n / q, q}(\Omega) \subset C^{s}(\Omega), \quad 0<s<1 .
\end{aligned}
$$

In order to get started, write

$$
p=\frac{1}{\gamma} \frac{n+2}{n-2}
$$

for some $\gamma>1$. Then for $f(u)$ as in (2.4.1),

$$
\begin{aligned}
u \in H_{\mathrm{loc}}^{1}(\Omega) & \Longrightarrow u \in L_{\mathrm{loc}}^{2 n /(n-2)}(\Omega) \quad(\text { if } n \geqslant 3) \\
& \Longrightarrow f(u) \in L_{\mathrm{loc}}^{2 n \gamma /(n+2)}(\Omega),
\end{aligned}
$$

so that (2.4.3) yields

$$
u \in H_{\mathrm{loc}}^{2,2 n \gamma /(n+2)}(\Omega)
$$

if $n \geqslant 3$. If $n=2$, then $u \in H_{\text {loc }}^{2, q}(\Omega)$ for all $q<\infty$. Observe

$$
\frac{2 n \gamma}{n+2}>\frac{n}{2} \Leftrightarrow \gamma>\frac{n+2}{4} \text {. }
$$

If (2.4.8) holds, we have

$$
u \in C^{s}(\Omega)
$$

for some $s \in(0,1)$. In the endpoint case $\gamma=(n+2) / 4$, we have

$$
u \in L_{\mathrm{loc}}^{q}(\Omega), \quad \forall q<\infty,
$$

and hence $f(u) \in L_{\text {loc }}^{q}(\Omega)$ for all $q<\infty$ as well. Then by (2.4.3),

$$
u \in H_{\mathrm{loc}}^{2, q}(\Omega), \quad \forall q<\infty,
$$


and (2.4.9) holds in this case as well. If $\gamma<(n+2) / 4$, we use the Sobolev embeddings (2.4.4) to get

$$
u \in L_{\mathrm{loc}}^{2 n \gamma /(n+2-4 \gamma)}(\Omega),
$$

and hence

$$
f(u) \in L_{\mathrm{loc}}^{2 n \gamma_{2} /(n+2)}(\Omega),
$$

where

$$
\gamma_{2}=\gamma^{2} \frac{n-2}{n+2-4 \gamma}>\gamma^{2} .
$$

Inserting this improved regularity for $f(u)$ into the elliptic regularity estimates (2.4.3) yields now

$$
u \in H_{\mathrm{loc}}^{2,2 n \gamma_{2} /(n+2)}(\Omega) .
$$

A finite number of iterations of this procedure yields the property (2.4.9). This in turn implies $f(u) \in C^{s}(\Omega)$, hence $u \in C^{s+2}(\Omega)$ for some $s \in(0,1)$.

From this, the conclusions of Proposition 2.4.1 follow, once we observe that if $p>1$ is not an odd integer, then one cannot improve the implication

$$
u \in C^{s}(\Omega), s \geqslant p, \Longrightarrow f(u) \in C^{p}(\Omega),
$$

except when $p$ is an even integer and $u$ does not change sign, while if $p$ is an odd integer, we get $f(u) \in C^{s}(\Omega)$.

For the rest of this section, we assume $M$ satisfies the weak homogeneity hypothesis. We want global estimates for functions $u \in H^{1}(M)$ satisfying (2.4.1) on all of $M$. We always have

$$
(\lambda-\Delta)^{-1}: H^{s, q}(M) \rightarrow H^{s+2, q}(M)
$$

whenever $\lambda>-\delta_{0}$ and $q=2$, however when $q \neq 2$ one often needs a stronger bound on $\lambda$. Hence we will take a different approach, which will also yield some decay estimates on solutions.

Let $\Omega \subset M$ be a bounded open set, $\widetilde{\Omega} \Subset \Omega$. Assume $\widetilde{\Omega}$ contains a ball of radius $D+1$. Then we can choose isometries $g_{j} \in G$ such that, if we set $\widetilde{\Omega}_{j}=g_{j}(\widetilde{\Omega})$, then the countable collection $\left\{\widetilde{\Omega}_{j}\right\}$ covers $M$, and we can assume (since $M$ has bounded geometry) that there exists $m<\infty$ such that each point $x \in M$ is in at most $m$ of the $\widetilde{\Omega}_{j}$.

Now depending on $p$ satisfying (1.0.9), let $L$ be the number of iterations required in the proof of Proposition 2.4.1. Choose intermediate nested open sets:

$$
\widetilde{\Omega} \Subset \Omega^{(L)} \Subset \cdots \Subset \Omega^{(1)} \Subset \Omega,
$$

along with the associated translates

$$
\Omega_{j}^{(\ell)}=g_{j}\left(\Omega^{(\ell)}\right) .
$$

We set

$$
A_{j}=\|u\|_{H^{1}\left(\Omega_{j}\right)}
$$

so that

$$
\|u\|_{H^{1}(M)}^{2} \simeq \sum_{j} A_{j}^{2}
$$


Applying the proof of Proposition 2.4.1 on $\Omega$ and its translates by isometries yields a similar statement on each $\Omega_{j}$ with constants independent of $j$. From (2.4.20), we have

$$
\|u\|_{L^{2 n /(n-2)}\left(\Omega_{j}\right)} \leqslant C_{1} A_{j}
$$

with $C_{1}$ independent of $j$. As usual, if $n=2$, this holds for $L^{q}\left(\Omega_{j}\right), q<\infty$. Hence

$$
\begin{aligned}
\|f(u)\|_{L^{2 n \gamma /(n+2)\left(\Omega_{j}\right)}} & \leqslant C_{2}\left\|u^{p}\right\|_{L^{2 n /(n-2) p}\left(\Omega_{j}\right)} \\
& \leqslant C_{2} C_{1}^{p} A_{j}^{p} .
\end{aligned}
$$

The local elliptic regularity then gives

$$
\|u\|_{H^{2,2 n \gamma /(n+2)\left(\Omega_{j}^{(1)}\right)}} \leqslant C_{3}\left(C_{2} C_{1}^{p} A_{j}^{p}+C_{1} A_{j}\right) \leqslant C_{4} A_{j} .
$$

Iterating this argument $L$ times, we obtain

$$
\|u\|_{C^{s}\left(\widetilde{\Omega}_{j}\right)} \leqslant C_{\star} A_{j}
$$

for a constant $C_{\star}$ independent of $j$, and where $s$ satisfies similar properties to that in Proposition 2.4.1] We record the result in the following Proposition.

Proposition 2.4.2. If $u \in H^{1}(M)$ is a solution to (2.4.1), then (2.4.25) holds with $A_{j}$ given by (2.4.20), $C_{\star}$ independent of $j$, and $s=2+p$ if $p \notin 2 \mathbb{N}$, and for every $s<2+p$ if $p \in 2 \mathbb{N}$.

Remark 2.4.3. Observe that the second inequality in (2.4.24) uses that $A_{j} \leqslant$ $\|u\|_{H^{1}(M)}$ for each $j$. Hence $C_{\star}$ is independent of $j$, but depends on $\|u\|_{H^{1}(M)}$ in a nonlinear fashion. In light of (2.4.21), we conclude

$$
\sum_{j}\|u\|_{C^{s}\left(\widetilde{\Omega}_{j}\right)}^{2} \leqslant C\left(\|u\|_{H^{1}(M)}\right)\|u\|_{H^{1}(M)}^{2} .
$$

2.5. Positivity of Minimizers. In this subsection we examine the question of positivity of minimizers. If $u \in H^{1}(M)$ is a minimizer of $F_{\lambda}$, subject to the constraint $J_{p}(u)=\beta$, set $v(x)=|u(x)|$. Of course we have

$$
\|v\|_{L^{2}}=\|u\|_{L^{2}}, \quad\|\nabla v\|_{L^{2}}=\|\nabla u\|_{L^{2}}, \quad\|v\|_{L^{p+1}}=\|u\|_{L^{p+1}},
$$

so $v$ is a solution to the same constrained minimization problem. But then

$$
v \geqslant 0, v \in H^{1}(M), \quad-\Delta v+\lambda v=K_{0}|v|^{p-1} v,
$$

with $K_{0}=I_{\beta} / \beta$ as in (1.1.5). Then Proposition 2.4.1 implies $v \in C^{2+p}(M)$, and $v \in C^{\infty}(M)$ if $p$ is an integer. We improve this in the next Proposition.

Proposition 2.5.1. The function $v=|u(x)|$ satisfies

$$
v(x)>0
$$

for all $x \in M$, and hence $v \in C^{\infty}(M)$.

This result is a consequence of the following Harnack inequality, which follows from Theorem 8.20 - Corollary 8.21 of [GT01. If $v \geq 0$ solves (2.5.2) on the weakly homogeneous space $M$, and if $\Omega_{j}^{(\ell)}$ are as in (2.4.18)-2.4.19), then (in light of the bounds on $|v|^{p-1}$ established in $\$ 2.4$ ) there exists a constant $C_{0}$, independent of $j$, such that

$$
\sup _{x \in \Omega_{j}^{(1)}} u(x) \leqslant C_{0} \inf _{x \in \Omega_{j}^{(1)}} u(x),
$$


Given (2.5.4), if $v \geq 0$ solves (2.5.2) and is not $\equiv 0$, strict positivity is immediate, and smoothness follows from Proposition 2.4.1, so Proposition 2.5.1 is proven. This in turn immediately gives the following.

Corollary 2.5.2. Every real-valued $F_{\lambda}$-minimizer $u$ satisfies either $u>0$ on $M$ or $u<0$ on $M$.

We now wish to extend the global regularity estimates on $u$ beyond $s=2+p$, when $u>0$ on $M$. The issue is that although $u>0$ on $M, u$ must decay at infinity, and since $f(u)$ is singular at $u=0$, there is some work to be done. Again the Harnack inequality (2.5.4) (with $u$ in place of $v$ ) provides the key to success. With this, we can establish the following improvement of Proposition 2.4.2.

Proposition 2.5.3. If $u \in H^{1}(M)$ solves (2.4.1) on $M$ and $u>0$, then (2.4.25) and (2.4.26) hold for every $s<\infty$ with constants depending on $s$ but not on $j$.

Proof. It suffices to prove the statment for $s \in \mathbb{N}$. Proposition 2.4 .2 implies that the assertions are true when $s=3$, and we proceed by induction. Let $k \in \mathbb{N}$ and suppose (2.4.25) holds for $s=k$. Then the covering property of the $\left\{\widetilde{\Omega}_{j}\right\}$ implies

$$
\|u\|_{C^{k}\left(\Omega_{j}\right)} \leqslant C_{k} A_{j}
$$

for some $C_{k}$ independent of $j$. We want to show

$$
\|u\|_{C^{k+1}\left(\widetilde{\Omega}_{j}\right)} \leqslant C_{k+1} A_{j}
$$

for some $C_{k+1}$ independent of $j$.

We need to estimate the $C^{k}$ norm of $f(u)$. The chain rule applied to $f(u)$ gives

$$
D^{\alpha} f(u)=\sum_{\gamma_{1}+\gamma_{2} \cdots+\gamma_{\nu}=\alpha, \nu,\left|\gamma_{\mu}\right| \geqslant 1} C_{\gamma} u^{\left(\gamma_{1}\right)} \cdots u^{\left(\gamma_{\nu}\right)} f^{(\nu)}(u) .
$$

Now

$$
\left|f^{(\nu)}(u)\right| \leqslant C|u|^{p-\nu},
$$

and from the Harnack inequality and our induction hypothesis, there exists $C_{1}, C_{2}$ such that

$$
C_{1} A_{j} \leqslant u \leqslant C_{2} A_{j} \text { on } \Omega_{j}^{(1)}
$$

so that

$$
\left|f^{(\nu)}\right| \leqslant C A_{j}^{p-\nu}
$$

Hence for $|\alpha| \leqslant k$ (so that, in particular, $\nu,\left|\gamma_{\mu}\right| \leqslant k$ ), we have

$$
\begin{aligned}
\left|D^{\alpha} f(u)\right| & \leqslant C \sum_{\gamma} A_{j}^{\nu} A_{j}^{p-\nu} \\
& \leqslant C^{\prime} A_{j}^{p} \\
& \leqslant C^{\prime \prime} A_{j}
\end{aligned}
$$

where again $C^{\prime \prime}$ is allowed to depend nonlinearly on the quantity $\|u\|_{H^{1}(M)}$. The last inequality in (2.5.11) uses the global bound $A_{j} \leq\|u\|_{H^{1}(M)}$. Hence

$$
\|f(u)\|_{C^{k}\left(\Omega_{j}^{(1)}\right)} \leqslant C A_{j} .
$$

Then the local elliptic regularity applied to (2.4.1) yields (2.5.6), completing the proof. 
2.6. Further decay estimates. In this section we continue to study properties of a positive solution $u \in H^{1}(M)$ to the elliptic equation

$$
-\Delta u+\lambda u=f(u),
$$

where

$$
f(u)=K|u|^{p-1} u .
$$

We also continue to assume this equation holds on a manifold $M$ that is weakly homogeneous in the sense described in the previous sections. So far we have shown that $u \in L^{q}(M)$ for every $q \in[2, \infty]$, and for each $s<\infty$,

$$
\|u\|_{C^{s}\left(\widetilde{\Omega}_{j}\right)} \leq C_{s} A_{j}, \quad \forall j,
$$

where $\left\{\widetilde{\Omega}_{j}\right\}$ is an open cover of $M$ by sets which are images under isometries of a fixed set $\widetilde{\Omega}$ and $A_{j}=\|u\|_{H^{1}\left(\Omega_{j}\right)}$, so

$$
\sum_{j} A_{j}^{2} \approx\|u\|_{H^{1}(M)}^{2} .
$$

These are varieties of decay results. In this section we seek stronger decay results. Here, we replace the hypothesis $\lambda>-\delta_{0}$ by

$$
\lambda>0,
$$

which for $\delta_{0}=0$ involves no change.

Since $\left\{e^{t \Delta}: t \geqslant 0\right\}$ is a contraction semigroup on $L^{q}(M)$ for each $q \geqslant 1$, we have

$$
(-\Delta+\lambda)^{-1}=\int_{0}^{\infty} e^{-\lambda t} e^{t \Delta} d t
$$

which implies

$$
(-\Delta+\lambda)^{-1}: L^{q}(M) \rightarrow L^{q}(M)
$$

for every $q \in[1, \infty]$, with operator norm bounded by $\lambda^{-1}$. Our previously estabished $L^{q}$ estimates on $u$ imply

$$
f(u) \in L^{q}(M), \quad \forall q \in[1, \infty] .
$$

Since $u=(-\Delta+\lambda)^{-1} f(u)$, we hence have $u \in L^{q}(M)$ for every $q \in[1, \infty]$.

Now set

$$
B_{j}=\|u\|_{L^{1}\left(\Omega_{j}\right)}
$$

so that

$$
\sum_{j} B_{j} \simeq\|u\|_{L^{1}(M)} .
$$

Comparing to (2.6.4), we see the collection $\left\{B_{j}\right\}$ satisfy "stricter bounds" than $\left\{A_{j}\right\}$. In this sense, the following result improves (2.6.3).

Proposition 2.6.1. For each $\epsilon \in(0,1)$, and each $s<\infty$, there exists $C_{\epsilon, s}<\infty$ such that

$$
\|u\|_{C^{s}\left(\widetilde{\Omega}_{j}\right)} \leqslant C_{\epsilon, s} A_{j}^{\epsilon} B_{j}^{1-\epsilon}, \quad \forall j .
$$


Proof. To start, (2.6.3) implies

$$
\|u\|_{L^{\infty}\left(\widetilde{\Omega}_{j}\right)} \leqslant C A_{j}
$$

for every $j$, with constants independent of $j$. Interpolating with (2.6.9), for each $\epsilon>0$ we can produce $q>1$ such that

$$
\|u\|_{L^{q}\left(\widetilde{\Omega}_{j}\right)} \leqslant C A_{j}^{\epsilon / 2} B_{j}^{1-\epsilon / 2},
$$

for every $j$, with constants indepenent of $j$. Next, (2.6.3) implies that for each $k<\infty$ there exists a constant $C_{k}<\infty$ such that

$$
\|u\|_{H^{k, q}\left(\tilde{\Omega}_{j}\right)} \leqslant C_{k} A_{j}
$$

for every $j$, where again $C_{k}$ is independent of $j$. Then interpolation with (2.6.13) implies that, for each $\epsilon \in(0,1), \sigma<\infty$, there exists $C_{\epsilon, \sigma}$ such that

$$
\|u\|_{H^{\sigma, q}\left(\widetilde{\Omega}_{j}\right)} \leqslant C_{\epsilon, \sigma} A_{j}^{\epsilon} B_{j}^{1-\epsilon},
$$

where again the constants are independent of $j$. Taking $\sigma>0$ sufficiently large proves the Proposition.

\section{Energy Minimizers}

In this section we tackle a different constrained minimization scheme, albeit for a slightly smaller range of powers $p$ in the nonlinear term of (1.0.1). The constrained minimization procedure is one that minimizes energy with respect to fixed mass. As stated in $\$ 1$, we now require

$$
p \in\left(1,1+\frac{4}{n}\right),
$$

which is the range of $L^{2}$ subcritical powers in the standard Euclidean case example. Such a case was also handled using concentration compactness on $\mathbb{R}^{n}$ in Lio84a, which we here generalize to the setting of weakly homogeneous spaces, $M$ as defined in 2.2 . We desire to minimize the functional

$$
E(u)=\frac{1}{2}\|\nabla u\|_{L^{2}}^{2}-\frac{1}{p+1} \int_{M}|u|^{p+1} d V
$$

over $H^{1}(M)$, subject to the constraint

$$
Q(u)=\|u\|_{L^{2}}^{2}=\beta .
$$

As in 92 , $H^{1}(M)$ will stand for $H_{0}^{1}(M)$ if $\partial M \neq \emptyset$ and we use the Dirichlet boundary condition. As seen in (1.2.1)-(1.2.3), given $u \in H^{1}(M)$ a solution to the constrained minimization problem, we must have, for some $\lambda \in \mathbb{R}$,

$$
-\Delta u+\lambda u-|u|^{p-1} u=0 .
$$

The range of powers in (3.0.1) plays an important role in the Gagliardo-Nirenberg inequality

$$
\|u\|_{L^{p+1}\left(\mathcal{R}^{n}\right)} \leqslant C\|u\|_{L^{2}}^{1-\gamma}\|u\|_{H^{1}}^{\gamma},
$$

where

$$
\gamma=\frac{n}{2}-\frac{n}{p-1}, \quad \text { hence } \gamma(p+1)<2
$$


As a result, we have

$$
\begin{aligned}
\|u\|_{H^{1}}^{2} & =E(u)+\frac{1}{p+1}\|u\|_{L^{p+1}}^{p+1}+Q(u) \\
& \leqslant E(u)+\tilde{C} Q(u)^{(p+1)(1-\gamma) / 2}\|u\|_{H^{1}}^{\gamma(p+1)}+Q(u),
\end{aligned}
$$

which gives a priori bounds at $\|u\|_{H^{1}}$ in terms of bounds on $E(u)$ and $Q(u)$. Then, as in (1.4.2), we take

$$
\mathcal{I}_{\beta}=\inf \left\{E(u): u \in H^{1}(M), Q(u)=\beta\right\},
$$

for $\beta>0$. The a priori bounds in (3.0.7) show that for a particular $\beta$, we have $\mathcal{I}_{\beta}>-\infty$ since $\gamma(p+1)<2$. Taking at this point the sequence $u_{\nu} \in H^{1}(M)$ such that

$$
Q\left(u_{\nu}\right)=\beta, E\left(u_{\nu}\right) \leqslant \mathcal{I}_{\beta}+\frac{1}{\nu} .
$$

Note, from (3.0.7), we have that $\left\|u_{\nu}\right\|_{H^{1}}$ bounded. Then, as in $\$ 2$, we apply the concentration-compactness techniques to the $L^{1}$ sequence given by $\left\{\left|u_{\nu}\right|^{2}\right\}$.

It turns out that we need to assume

$$
\mathcal{I}_{\beta}<0,
$$

to show in the concentration-compactness argument that splitting and vanishing cannot occur. In connection with this, note that replacing $u$ by $a u$ in (3.0.2) and letting $a \nearrow+\infty$ shows that

$$
\mathcal{I}_{\beta} \rightarrow-\infty \text { as } \beta \rightarrow+\infty .
$$

In particular, $\mathcal{I}_{\beta}<0$ for all sufficiently large $\beta$. However, it is not guaranteed that $\mathcal{I}_{\beta}<0$ for all $\beta$. See Appendix A.3 for more on this. Exploration of when such a negative energy condition is satisfied for a weakly homogeneous space is an interesting area for future research.

In $\$ 3.1$ we demonstrate concentration for a subsequence of a minimizing sequence (3.0.9), when (3.0.10) holds. In $\$ 3.2$ we establish compactness and prove existence of energy minimizers when $M$ is a weakly homogeneous space, again under the hypothesis (3.0.10). (If $M$ is compact, (3.0.10) is not needed.) In 33.3 we show that the examples of manifolds with no $F_{\lambda}$-minimizers given in $\$ 2.3$ also have no energy minimizers. In $\$ 3.4$ we note how results on smoothness, positivity, and decay established for $F_{\lambda}$-minimizers in $\S \$ 2.42 .6$ also hold for energy minimizers. In $\$ 3.5$ we compute the second variation of energy for an energy minimizer, expressed in terms of operators $L_{ \pm}$, defined in (3.5.42). In 3.6 we give similar formulas for the second variation of $F_{\lambda}$, for $F_{\lambda}$-minimizers. In 33.7 we examine some spectral properties of $L_{ \pm}$, and draw a number of conclusions. In particular, we deduce from the fact that $L_{-} \geqslant 0$ that whenever an energy minimizer $u \in H^{1}(M)$ satisfies (3.0.4), $\operatorname{Spec}(-\Delta+\lambda) \subset[0, \infty)$. Results of $\$ 3.7$ are applied in $\$ 3.8$ to results concerning orbital stability.

3.1. Concentration. We need to show that there is no vanishing and no splitting. We first establish that there is no vanishing when $\mathcal{I}_{\beta}<0$. In fact, The vanishing condition for our sequence implies that given $B_{R}(y)=\left\{x \in M: d_{M}(x, y) \leqslant R\right\}$, we have

$$
\lim _{\nu \rightarrow \infty} \sup _{y \in M} \int_{B_{R}(y)}\left|u_{\nu}\right|^{2} d V=0, \quad \forall R<\infty .
$$


Using Lemma 2.1.2 we have the following.

Proposition 3.1.1. Assume $\mathcal{I}_{\beta}<0$. For $u_{\nu}$ a sequence minimizing the energy with fixed mass, (3.1.1) cannot occur.

Proof. For $p$ as in (3.0.1), let us assume that (3.1.1) occurs for $\left\{u_{\nu}\right\}$. Then, Lemma 2.1 .2 shows

$$
\left\|u_{\nu}\right\|_{L^{p+1}} \rightarrow 0
$$

implying

$$
\frac{1}{2}\left\|\nabla u_{\nu}\right\|_{L^{2}}^{2} \rightarrow \mathcal{I}_{\beta}<0
$$

a contradiction.

Our next task is to establish that there is no splitting. If there were splitting, we see that for any $\alpha \in(0, \beta)$, for each $\epsilon>0$ there exists $\nu_{0} \geqslant 1$ and sets $E_{\nu}^{\#}, E_{\nu}^{b} \subset M$ such that

$$
d\left(E_{\nu}^{\#}, E_{\nu}^{b}\right) \rightarrow \infty \text { as } \nu \rightarrow \infty
$$

and

$$
\left.\left|\int_{E_{\nu}^{\#}}\right| u_{\nu}\right|^{2} d V-\alpha|<\epsilon,| \int_{E_{\nu}^{b}}\left|u_{\nu}\right|^{2} d V-(\beta-\alpha) \mid<\epsilon .
$$

We record here some subadditivity properties of $\mathcal{I}_{\beta}$ in order to argue similarly to the splitting argument in Section 2.1

Proposition 3.1.2. If $\beta>0, \mathcal{I}_{\beta}<0, \sigma>1$, then

$$
\mathcal{I}_{\sigma \beta}<\sigma \mathcal{I}_{\beta} .
$$

Proof. Let $u_{\nu}$ be a minimizing sequence as in (3.0.9). Define

$$
w_{\nu}=\sigma^{1 / 2} u_{\nu}, \quad \text { so }\left\|w_{\nu}\right\|_{L^{2}}^{2}=\sigma \beta .
$$

Hence,

$$
\begin{aligned}
E\left(w_{\nu}\right) & =\frac{\sigma}{2}\left\|\nabla u_{\nu}\right\|_{L^{2}}^{2}-\frac{\sigma^{p+1}}{p+1} \int_{M}\left|u_{\nu}\right|^{p+1} d V \\
& =\sigma E\left(u_{\nu}\right)-\frac{\sigma^{p+1}-\sigma}{p+1}\left\|u_{\nu}\right\|_{L^{p+1}}^{p+1} .
\end{aligned}
$$

Passing to the limit gives

$$
\mathcal{I}_{\sigma \beta} \leqslant \sigma \mathcal{I}_{\beta} .
$$

However, given $\mathcal{I}_{\beta}<0$, as in the proof of Proposition 3.1.1 $\left\|u_{\nu}\right\|_{L^{p+1}}$ does not approach 0 and the result follows.

Then, we have the following result similar to Proposition 2.1.4.

Proposition 3.1.3. Given $0<\eta<\beta$ and $\mathcal{I}_{\beta}<0$, we have

$$
\mathcal{I}_{\beta}<\mathcal{I}_{\beta-\eta}+\mathcal{I}_{\eta} \text {. }
$$


Proof. Let us assume without loss of generality that $\eta \leqslant \beta-\eta$ and take

$$
\beta-\eta=\sigma \eta
$$

with $\sigma \geqslant 1$. Hence, using Proposition 3.1 .2 we have

$$
\mathcal{I}_{\sigma \eta} \leqslant \sigma \mathcal{I}_{\eta}, \mathcal{I}_{\beta}=\mathcal{I}_{(\sigma+1) \eta}<\frac{\sigma+1}{\sigma} \mathcal{I}_{\sigma \eta}=\frac{\sigma+1}{\sigma} \mathcal{I}_{\beta-\eta} .
$$

As a result,

$$
\begin{aligned}
\mathcal{I}_{\beta}=\mathcal{I}_{(\sigma+1) \eta} & <\frac{\sigma+1}{\sigma} \mathcal{I}_{\sigma \eta} \\
& =\mathcal{I}_{\beta-\eta}+\frac{1}{\sigma} \mathcal{I}_{\sigma \eta} \\
& \leqslant \mathcal{I}_{\beta-\eta}+\mathcal{I}_{\eta} .
\end{aligned}
$$

Applying this proposition in the same way as in Section 2.1, we have the result that no splitting can occur for the sequence $\left\{u_{\nu}\right\}$.

Therefore, upon passing to a subsequence, we have concentration. There exist $y_{\nu} \in M$ (independent of $\epsilon$ ) with the following property. For each $\epsilon>0$, there exists $\widetilde{R}(\epsilon)<\infty$ such that

$$
\int_{B_{\widetilde{R}(\epsilon)}\left(y_{\nu}\right)}\left|u_{\nu}\right|^{2} d V>\beta-\epsilon .
$$

\subsection{Existence of energy minimizers. As long as}

$$
\mathcal{I}_{\beta}<0,
$$

we are left with the situation where $u_{\nu} \in H^{1}(M)$ satisfies (3.0.9) and the concentration phenomenon (3.1.14). If $M$ is weakly homogeneous, we can translate the points $y_{\nu}$ to a subset of some compact $K \subset M$. We relabel the associated translates of $u_{\nu}$ as $u_{\nu}$. Passing to a subsequence, we have

$$
u_{\nu} \longrightarrow u, \quad \text { weak }^{*} \text { in } H^{1}(M) .
$$

By Rellich compactness, $u_{\nu} \rightarrow u$ in $L^{2}(B)$, in norm, for each bounded $B \subset M$. Hence, by (3.1.14)

$$
\|u\|_{L^{2}}^{2}=\beta
$$

Hence,

$$
u_{\nu} \longrightarrow u \text { in } L^{2}(M) \text { norm. }
$$

Now, as in (3.0.5), we have

$\mathrm{SO}$

$$
\left\|u-u_{\nu}\right\|_{L^{p+1}} \leq C\left\|u-u_{\nu}\right\|_{L^{2}}^{1-\gamma}\left\|u-u_{\nu}\right\|_{H^{1}}^{\gamma},
$$

Now

$$
u_{\nu} \longrightarrow u \text { in } L^{p+1}(M) \text { norm. }
$$

and

$$
\frac{1}{2}\left\|\nabla u_{\nu}\right\|_{L^{2}}^{2}-\frac{1}{p+1}\left\|u_{\nu}\right\|_{L^{p+1}}^{p+1} \longrightarrow \mathcal{I}_{\beta},
$$

$$
\left\|u_{\nu}\right\|_{L^{p+1}}^{p+1} \longrightarrow\|u\|_{L^{p+1}}^{p+1} .
$$


Also, since (3.2.3) holds,

$$
\frac{1}{2}\|\nabla u\|_{L^{2}}^{2}-\frac{1}{p+1}\|u\|_{L^{p+1}}^{p+1} \geq \mathcal{I}_{\beta} .
$$

Hence

$$
\|\nabla u\|_{L^{2}}^{2} \geq \liminf \left\|\nabla u_{\nu}\right\|_{L^{2}}^{2}
$$

So

$$
\nabla u_{\nu} \longrightarrow \nabla u \text { in } L^{2}(M) \text { norm, }
$$

and $u$ minimizes $E(u)$ subject to the constraint (3.0.3), at least provided (3.2.1) holds.

Here is one basic case where the hypothesis (3.2.1) can be removed.

Proposition 3.2.1. Let $M$ be a compact $n$-dimensional Riemannian manifold, possibly with boundary, and assume $p$ satisfies 3.0.1). Then, given $\beta>0$, there exists $u \in H^{1}(M)$ such that $Q(u)=\beta$ and $E(u)=\mathcal{I}_{\beta}$.

Proof. Taking $u_{\nu}$ as in (3.0.9), we have a bound on $\left\|u_{\nu}\right\|_{H^{1}}$. If $u_{\nu} \rightarrow u$ weak $^{*}$ in $H^{1}(M)$, the Rellich compactness theorem yields $u_{\nu} \rightarrow u$ in norm in both $L^{2}(M)$ and $L^{p+1}(M)$. Hence $Q(u)=\beta$ and $\|u\|_{L^{p+1}}=\lim \left\|u_{\nu}\right\|_{L^{p+1}}$, hence $E(u) \leq \lim E\left(u_{\nu}\right)$. This implies $E(u)=\mathcal{I}_{\beta}$ (and also $u_{\nu} \rightarrow u$ in $H^{1}$-norm).

3.3. Manifolds with no energy minimizers. Here we show that the manifolds with no $F_{\lambda}$-minimizers exhibited in $\$ 2.3$ also have no energy minimizers. Since the arguments are similar, we will be brief. Let $M=\mathbb{R}^{n} \backslash K$, where $K \subset \mathbb{R}^{n}$ is a smoothly bounded, compact set. We impose the Dirichlet boundary condition on $\partial M$, take $\beta>0$, and ask whether we can minimize $E(u)$, given

$$
u \in H_{0}^{1}(M), \quad Q(u)=\beta .
$$

We will show that no such minimizer exists. To see this, set

$$
\mathcal{I}_{\beta}(M)=\inf \left\{E(u): u \in H_{0}^{1}(M), Q(u)=\beta\right\} .
$$

It is clear that $\mathcal{I}_{\beta}(M) \geq \mathcal{I}_{\beta}\left(\mathbb{R}^{n}\right)$, since $H_{0}^{1}(M) \subset H^{1}\left(\mathbb{R}^{n}\right)$. An argument similar to that in $\$ 2.3$ yields the reverse inequality, so

$$
\mathcal{I}_{\beta}(M)=\mathcal{I}_{\beta}\left(\mathbb{R}^{n}\right) .
$$

The positivity results of 2.5 apply to this setting (for more on this, see 33.4 ). The proof of Proposition 2.3.1 is hence readily modified, to yield:

Proposition 3.3.1. If $M=\mathbb{R}^{n} \backslash K$, there does not exist $u \in H_{0}^{1}(M)$ such that $Q(u)=\beta$ and $E(u)=\mathcal{I}_{\beta}(M)$.

As in 2.3 we can replace $\mathbb{R}^{n}$ by a general noncompact, connected, weakly homogeneous space and get a similar nonexistence result.

3.4. Smoothness, positivity, and decay of energy minimizers. In this brief section, we make note of how results of $\S \$ 2.42 .6$ apply to energy minimizers. We return to the setting where $M$ is a weakly homogeneous. As was done in $\S \$ 2.42 .6$, in this section we assume, for the sake of simplicity, that $\partial M=\emptyset$.

If $u \in H^{1}(M)$ minimizes (3.0.2), subject to the constraint (3.0.3), so does $v=|u|$, so $v$ solves

$$
v \in H^{1}(M), v \geq 0, \quad-\Delta v+\lambda v-|v|^{p-1} v=0,
$$


for some $\lambda \in \mathbb{R}$. Boundedness (and decay) results of 2.4 hold. Then, as in 22.5 , the Harnack inequality (2.5.4) impliies $v>0$ on $M$, and we get:

Proposition 3.4.1. Every real-valued energy minimizer $u$ satisfies either $u>0$ on $M$ or $u<0$ on $M$, and belongs to $\mathcal{C}^{\infty}(M)$.

Given this, the decay results Proposition 2.5.3 and Proposition 2.6.1 apply to these energy minimizers.

3.5. Second variation of energy. With $\beta \in(0, \infty)$, let

$$
X=\left\{u \in H^{1}(M): Q(u)=\beta\right\}, \quad \mathcal{I}_{\beta}=\inf \{E(u): u \in X\},
$$

and

$$
Y=\left\{u \in X: E(u)=\mathcal{I}_{\beta}\right\} .
$$

Conditions guaranteeing that $Y$ is nonempty have been given in $\$ 3.2$. Recall that $E(u)$ and $Q(u)$ are given by (3.0.2)- (3.0.3). Here we study

$$
\frac{d^{2}}{d s^{2}} E(w(s))
$$

when $w(s)$ is a smooth path in $X$ satisfying $w(0)=u \in Y$. To be definite, take $u \in Y$,

$$
\psi \in T_{u} X=\left\{\psi \in H^{1}(M): \operatorname{Re}(u, \psi)=0\right\}
$$

and set

$$
w(s)=a \frac{u+s \psi}{\|u+s \psi\|}, \quad a=\beta^{1 / 2} .
$$

In light of the discussion in 3.4 we can assume

$$
u>0 \text { on } M,
$$

but we cannot assume $\psi$ is real valued. Set

$$
\psi=\psi_{0}+i \psi_{1}, \quad \psi_{0}, \psi_{1} \text { real valued. }
$$

Then the condition (3.5.4) is equivalent to

$$
\left(u, \psi_{0}\right)=0
$$

with no constraint on $\psi_{1}$.

The chain rule gives

$$
\frac{d}{d s} E(w(s))=D E(w(s)) w^{\prime}(s),
$$

and in particular

$$
\left.\frac{d}{d s} E(w(s))\right|_{s=0}=D E(u) w^{\prime}(0) .
$$

Differentiating (3.5.9) gives

$$
\frac{d^{2}}{d s^{2}} E(w(s))=D^{2} E(w(s))\left(w^{\prime}(s), w^{\prime}(s)\right)+D E(w(s)) w^{\prime \prime}(s),
$$

and in particular

$$
\left.\frac{d^{2}}{d s^{2}} E(w(s))\right|_{s=0}=D^{2} E(u)\left(w^{\prime}(0), w^{\prime}(0)\right)+D E(u) w^{\prime \prime}(0) .
$$


We turn to the computation of $w^{\prime}(0)$ and $w^{\prime \prime}(0)$. Rewrite 3.5.5) as

$$
\begin{aligned}
& w(s)=a F(s)(u+s \psi), \\
& F(s)=\|u+s \psi\|^{-1}=\left(a^{2}+\|\psi\|^{2}\right)^{-1 / 2} .
\end{aligned}
$$

Then

$$
F^{\prime}(s)=-s\left(a^{2}+s^{2}\|\psi\|^{2}\right)^{-3 / 2}\|\psi\|^{2},
$$

$$
F^{\prime \prime}(s)=-\left(a^{2}+s^{2}\|\psi\|^{2}\right)^{-3 / 2}\|\psi\|^{2}-s\|\psi\|^{2} \frac{d}{d s}\left(a^{2}+s^{2}\|\psi\|^{2}\right)^{-3 / 2},
$$

SO

$$
F^{\prime}(0)=0, \quad F^{\prime \prime}(0)=-a^{-3}\|\psi\|^{2} .
$$

We have

$$
\begin{aligned}
& w^{\prime}(s)=a F(s) \psi+a F^{\prime}(s)(u+s \psi), \quad \text { so } \\
& w^{\prime}(0)=a F(0) \psi=\psi,
\end{aligned}
$$

and

$$
\begin{aligned}
& w^{\prime \prime}(s)=2 a F^{\prime}(s) \psi+a F^{\prime \prime}(s) u, \quad \text { so } \\
& w^{\prime \prime}(0)=a F^{\prime \prime}(0) u=-\frac{\|\psi\|^{2}}{a^{2}} u .
\end{aligned}
$$

Thus (3.5.10) and (3.5.12) become

$$
\left.\frac{d}{d s} E(w(s))\right|_{s=0}=D E(u) \psi,
$$

and

$$
\left.\frac{d^{2}}{d s^{2}} E(w(s))\right|_{s=0}=D^{2} E(u)(\psi, \psi)-\frac{1}{a^{2}}\|\psi\|^{2} D E(u) u
$$

Also

$$
D E(u) \psi=\left.\frac{d}{d s} E(u+s \psi)\right|_{s=0},
$$

and

$$
D^{2} E(u)(\psi, \psi)=\left.\frac{d^{2}}{d s^{2}} E(u+s \psi)\right|_{s=0} .
$$

Our next task is to compute the right sides of (3.5.20) and (3.5.21). It is convenient to set

$$
E(u)=T(u)-\widetilde{J}(u)
$$

with

$$
T(u)=\frac{1}{2}\|\nabla u\|_{L^{2}}^{2}, \quad \widetilde{J}(u)=\frac{1}{p+1}\|u\|_{L^{p+1}}^{p+1} .
$$

First, the calculation

$$
\begin{aligned}
T(s+s \psi) & =\frac{1}{2}\|\nabla u+s \nabla \psi\|^{2} \\
& =\frac{1}{2}\|\nabla u\|^{2}+s \operatorname{Re}(\nabla u, \nabla \psi)+\frac{s^{2}}{2}\|\nabla \psi\|^{2}
\end{aligned}
$$

gives

$$
\left.\frac{d}{d s} T(u+s \psi)\right|_{s=0}=\operatorname{Re}(\nabla u, \nabla \psi)=\operatorname{Re}(-\Delta u, \psi)
$$


and

$$
\left.\frac{d^{2}}{d s^{2}} T(u+s \psi)\right|_{s=0}=\|\nabla \psi\|^{2}=\left\|\nabla \psi_{0}\right\|^{2}+\left\|\nabla \psi_{1}\right\|^{2} .
$$

Next, write

$$
\widetilde{J}(u+s \psi)=\frac{1}{p+1} \int_{M}(u+s \psi)^{(p+1) / 2}(\bar{u}+s \bar{\psi})^{(p+1) / 2} d V .
$$

Then

$$
\begin{aligned}
\frac{d}{d s} \widetilde{J}(u+s \psi)=\frac{1}{2} \int_{M}[ & (u+s \psi)^{(p-1) / 2}(\bar{u}+s \bar{\psi})^{(p+1) / 2} \psi \\
& \left.+(u+s \psi)^{(p+1) / 2}(\bar{u}+s \bar{\psi})^{(p-1) / 2} \bar{\psi}\right] d V
\end{aligned}
$$

and

$$
\begin{aligned}
\frac{d^{2}}{d s^{2}} \widetilde{J}(u+s \psi)=\frac{1}{2} \int_{M}[ & \frac{p+1}{2}(u+s \psi)^{(p-1) / 2}(\bar{u}+s \bar{\psi})^{(p-1) / 2} \psi \bar{\psi} \\
& +\frac{p-1}{2}(u+s \psi)^{(p-3) / 2}(\bar{u}+s \bar{\psi})^{(p+1) / 2} \psi^{2} \\
& +\frac{p+1}{2}(u+s \psi)^{(p-1) / 2}(\bar{u}+s \bar{\psi})^{(p-1) / 2} \psi \bar{\psi} \\
& \left.+\frac{p-1}{2}(u+s \psi)^{(p+1) / 2}(\bar{u}+s \bar{\psi})^{(p-3) / 2} \bar{\psi}^{2}\right] d V .
\end{aligned}
$$

In particular,

$$
\begin{aligned}
\left.\frac{d}{d s} \widetilde{J}(u+s \psi)\right|_{s=0} & =\frac{1}{2} \int_{M}|u|^{p-1}(\bar{u} \psi+u \bar{\psi}) \\
& =\operatorname{Re}\left(|u|^{p-1} u, \psi\right) .
\end{aligned}
$$

Before evaluating (3.5.29) at $s=0$, let us record that (3.5.25) and (3.5.30) imply

$$
\left.\frac{d}{d s} E(u+s \psi)\right|_{s=0}=\operatorname{Re}\left(-\Delta u-|u|^{p-1} u, \psi\right) .
$$

(This calculation does not use (3.5.6).) For $u \in Y$, i.e., a minimizer of $\left.E\right|_{X}$, this must vanish for all $\psi \in T_{u} X$, described by (3.5.4). Consequently, given $u \in Y$,

$$
\psi \in H^{1}(M), \operatorname{Re}(u, \psi)=0 \Longrightarrow \operatorname{Re}\left(\Delta u+|u|^{p-1} u, \psi\right)=0 .
$$

It follows that there exists $\lambda \in \mathbb{R}$ such that

$$
\Delta u+|u|^{p-1} u=\lambda u,
$$

and we recover (1.2.2)-11.2.3).

We also note that the last term in (3.5.19) is

$$
\begin{aligned}
\frac{1}{a^{2}}\|\psi\|^{2} \frac{d}{d s} & \left.E(u+s u)\right|_{s=0} \\
& =\frac{1}{a^{2}}\|\psi\|^{2} \operatorname{Re}\left(-\Delta u-|u|^{p-1} u, u\right) \\
& =\frac{1}{a^{2}}\|\psi\|^{2}(-\lambda u, u) \\
& =-\lambda\|\psi\|^{2},
\end{aligned}
$$


the second identity by (3.5.33).

We now evaluate (3.5.29) at $s=0$. For this, we will use (3.5.6), and write $\psi=\psi_{0}+i \psi_{1}$, as in (3.5.7). We have

$$
\begin{aligned}
\left.\frac{d^{2}}{d s^{2}} \widetilde{J}(u+s \psi)\right|_{s=0}= & \frac{1}{2} \int_{M}\left[(p+1)|u|^{p-1}|\psi|^{2}\right. \\
& \quad+\frac{p-1}{2} u^{(p-3) / 2} \bar{u}^{(p+1) / 2} \psi^{2} \\
& \left.\quad+\frac{p-1}{2} u^{(p+1) / 2} \bar{u}^{(p-3) / 2} \bar{\psi}^{2}\right] d V \\
= & \frac{1}{2}\left((p+1)|u|^{p-1} \psi, \psi\right)+\frac{p-1}{2} \operatorname{Re}\left(|u|^{p-3} u^{2} \bar{\psi}, \psi\right) \\
= & \frac{p+1}{2}\left(u^{p-1} \psi, \psi\right)+\frac{p-1}{2} \operatorname{Re}\left(u^{p-1} \bar{\psi}, \psi\right),
\end{aligned}
$$

the last identity by 3.5 .6 . Now

$$
\left(u^{p-1} \psi, \psi\right)=\left(u^{p-1} \psi_{0}, \psi_{0}\right)+\left(u^{p-1} \psi_{1}, \psi_{1}\right)
$$

and

$$
\begin{aligned}
\operatorname{Re}\left(u^{p-1} \bar{\psi}, \psi\right) & =\operatorname{Re} \int_{M} u^{p-1}\left(\psi_{0}-i \psi_{1}\right)^{2} d V \\
& =\left(u^{p-1} \psi_{0}, \psi_{0}\right)-\left(u^{p-1} \psi_{1}, \psi_{1}\right)
\end{aligned}
$$

SO

$$
\begin{aligned}
\left.\frac{d^{2}}{d s^{2}} \widetilde{J}(u+s \psi)\right|_{s=0}= & \frac{p+1}{2}\left(u^{p-1} \psi_{0}\right)+\frac{p-1}{2}\left(u^{p-1} \psi_{0}, \psi_{0}\right) \\
& +\frac{p+1}{2}\left(u^{p-1} \psi_{1}, \psi_{1}\right)-\frac{p-1}{2}\left(u^{p-1} \psi_{1}, \psi_{1}\right) \\
= & p\left(u^{p-1} \psi_{0}, \psi_{0}\right)+\left(u^{p-1} \psi_{1}, \psi_{1}\right) .
\end{aligned}
$$

Together with (3.5.26), this gives

$$
\begin{aligned}
\left.\frac{d^{2}}{d s^{2}} E(u+s \psi)\right|_{s=0}= & \left(-\Delta \psi_{0}-p u^{p-1} \psi_{0}, \psi_{0}\right) \\
& +\left(-\Delta \psi_{1}-u^{p-1} \psi_{1}, \psi_{1}\right)
\end{aligned}
$$

This, together with (3.5.19) and (3.5.34), yields

$$
\begin{aligned}
\left.\frac{d^{2}}{d s^{2}} E(w(s))\right|_{s=0}= & \left(\left(-\Delta-p u^{p-1}\right) \psi_{0}, \psi_{0}\right)+\lambda\left(\psi_{0}, \psi_{0}\right) \\
& +\left(\left(-\Delta-u^{p-1}\right) \psi_{1}, \psi_{1}\right)+\lambda\left(\psi_{1}, \psi_{1}\right),
\end{aligned}
$$

when $w(s)$ is given by (3.5.5). In other words,

$$
\left.\frac{d^{2}}{d s^{2}} E(w(s))\right|_{s=0}=\left(L_{+} \psi_{0}, \psi_{0}\right)+\left(L_{-} \psi_{1}, \psi_{1}\right),
$$

with $L_{ \pm}: H^{1}(M) \rightarrow H^{-1}(M)$ given by

$$
\begin{aligned}
& L_{+} \psi_{0}=\left(-\Delta+\lambda-p|u|^{p-1}\right) \psi_{0}, \\
& L_{-} \psi_{1}=\left(-\Delta+\lambda-|u|^{p-1}\right) \psi_{1} .
\end{aligned}
$$

The Friedrichs method defines $L_{+}$and $L_{-}$as self-adjoint operators on $L^{2}(M)$, with domain $H^{2}(M)$. 
3.6. Second variation of $F_{\lambda}$. In this setting, we take $p$ as in (1.0.9), $J_{p}$ as in (1.0.8), and $F_{\lambda}$ as in (1.0.7). With $\beta \in(0, \infty)$, set

$$
\widetilde{X}=\left\{u \in H^{1}(M): J_{p}(u)=\beta\right\}, \quad I_{\beta}=\inf \left\{F_{\lambda}(u): u \in \widetilde{X}\right\},
$$

and

$$
\widetilde{Y}=\left\{u \in \widetilde{X}: F_{\lambda}(u)=I_{\beta}\right\} .
$$

Conditions guaranteeing that $\tilde{Y}$ is nonempty have been given in $\oint_{2}$, Here we compute

$$
\frac{d^{2}}{d s^{2}} F_{\lambda}(w(s))
$$

where $w(s)$ is a smooth path in $\widetilde{X}$ satisfying $w(0)=u \in \widetilde{Y}$. To be definite, take $u \in \widetilde{Y}$ (we can and will assume $u>0$ ), take

$$
\psi \in T_{u} \tilde{X}=\left\{\psi \in H^{1}(M): \operatorname{Re}\left(u^{p}, \psi\right)=0\right\},
$$

and set

$$
w(s)=a \frac{u+s \psi}{\|u+s \psi\|_{L^{p+1}}}, \quad a=\|u\|_{L^{p+1}}=\beta^{1 /(p+1)} .
$$

A calculation parallel to that done for $(d / d s)^{2} E(w(s))$ in 33.5 , which this time we leave to the reader, gives

$$
\left.\frac{1}{2} \frac{d^{2}}{d s^{2}} F_{\lambda}(w(s))\right|_{s=0}=\left(L_{+} \psi_{0}, \psi_{0}\right)+\left(L_{-} \psi_{1}, \psi_{1}\right),
$$

where $\psi=\psi_{0}+i \psi_{1}$, with $\psi_{0}, \psi_{1}$ real valued, and $L_{ \pm}$as in (3.5.42), i.e.,

$$
\begin{aligned}
& L_{+} \psi_{0}=\left(-\Delta+\lambda-p|u|^{p-1}\right) \psi_{0}, \\
& L_{-} \psi_{1}=\left(-\Delta+\lambda-|u|^{p-1}\right) \psi_{1},
\end{aligned}
$$

provided a certain rescaling, described below, is performed. In this case, the condition that $\psi \in H^{1}(M)$ belong to $T_{u} \widetilde{X}$ becomes

$$
\left(u^{p}, \psi_{0}\right)=0,
$$

with no further condition on $\psi_{1}$. Contrast (3.6.8) with (3.5.8).

We describe the rescaling of $u$ that yields (3.6.7). If $\widetilde{X}$ is as in (3.6.1) and if $u \in \widetilde{Y}$ as in (3.6.2) and is $\geqslant 0$, then there exists $K \in \mathbb{R}$ such that such that

$$
-\Delta u+\lambda u=K u^{p} \text {. }
$$

Taking the inner product with $u$ yields

$$
K=\beta^{-1} I_{\beta} .
$$

We can rescale, replacing $u$ by $\kappa u$, to arrange that $K=1$ in (3.6.9), so, with a different $\beta$, the new $u$ minimizes $F_{\lambda}$ subject to the constraint $J_{p}(u)=\beta$, and satisfies

$$
-\Delta u+\lambda u-u^{p}=0 .
$$

Cf. Corollary 2.1.5. It is for this rescaled $u$ that (3.6.6)-3.6.7) hold. 
3.7. Properties of $L_{ \pm}$. Throughout this subsection, $M$ will be a weakly homogeneous space. We have defined operators $L_{+}$and $L_{-}$in $\left.\S \$ 3.5\right] 3.6$, as

$$
\begin{aligned}
& L_{+} \psi_{0}=\left(-\Delta+\lambda-p|u|^{p-1}\right) \psi_{0}, \\
& L_{-} \psi_{1}=\left(-\Delta+\lambda-|u|^{p-1}\right) \psi_{1},
\end{aligned}
$$

arising when $u \in H^{1}(M)$ is either an energy minimizer of an $F_{\lambda}$-monimizer, satisfying (3.6.11). We also assume $u>0$. In light of decay results on $u$ established in $₫ 2$, these are self-adjoint operators on $L^{2}(M)$, with domain $H^{2}(M)$. In these respective cases, we have seen that, with $w(s)$ respectively as in (3.5.5) or (3.6.5),

$$
\begin{gathered}
\left.\frac{d^{2}}{d s^{2}} E(w(s))\right|_{s=0}=\left(L_{+} \psi_{0}, \psi_{0}\right)+\left(L_{-} \psi_{1}, \psi_{1}\right), \\
\left.\frac{d^{2}}{d s^{2}} F_{\lambda}(w(s))\right|_{s=0}=\left(L_{+} \psi_{0}, \psi_{0}\right)+\left(L_{-} \psi_{1}, \psi_{1}\right),
\end{gathered}
$$

with $\psi_{j} \in H^{1}(M)$ real valued, $\psi_{1}$ otherwise arbitrary, and

$$
\begin{aligned}
& \left(u, \psi_{0}\right)=0 \text { in case (3.7.2), } \\
& \left(u^{p}, \psi_{0}\right)=0 \text { in case 3.7.3). }
\end{aligned}
$$

Since $u$ is a minimizer, we know that (3.7.2) (resp., (3.7.3)) is $\geqslant 0$ for all such paths $w(s)$. We deduce that

$$
\left(L_{+} \psi_{0}, \psi_{0}\right) \geqslant 0,
$$

for all real-valued $\psi_{0} \in H^{1}(M)$ satisfying (3.7.4) when $u$ is an energy minimizer, and for all $\psi_{0}$ satisfying (3.7.5) when $u$ is an $F_{\lambda}$-minimizer (satisfying (3.6.11)). Also, in both cases,

$$
\left(L_{-} \psi_{1}, \psi_{1}\right) \geqslant 0,
$$

for all real valued $\psi_{1} \in H^{1}(M)$. Since $L_{+}$and $L_{-}$are reality preserving, these results extend readily to the case where $\psi_{0}$ and $\psi_{1}$ are allowed to be complex valued.

As we have seen, if $u \in H^{1}(M)$ minimizes $E(u)$, subject to the constraint $\|u\|_{L^{2}}^{2}=\beta$, then there exists $\lambda \in \mathbb{R}$ such that

$$
\Delta u-\lambda u+|u|^{p-1} u=0 .
$$

(This is also the PDE satisfied by the rescaled $F_{\lambda}$-minimizer, discussed in $\$ 3.6$.) From (3.7.7), we have the following information about $\lambda$.

Proposition 3.7.1. If $u$ is an energy minimizer satisfying (3.7.8), then

$$
\operatorname{Spec}(-\Delta+\lambda) \subset[0, \infty) \text {. }
$$

Proof. In fact, for all $\psi \in H^{1}(M)$,

$$
\begin{aligned}
((-\Delta+\lambda) \psi, \psi) & =\left(L_{-} \psi, \psi\right)+\left(|u|^{p-1} \psi, \psi\right) \\
& \geqslant\left(|u|^{p-1} \psi, \psi\right) \\
& \geqslant 0 .
\end{aligned}
$$


By contrast with the positivity of $L_{-}$, note that

$$
\begin{aligned}
\left(L_{+} u, u\right) & =-\left(\Delta u-\lambda u+p|u|^{p-1} u, u\right) \\
& =-(p-1) \int_{M}|u|^{p+1} d V \\
& <0,
\end{aligned}
$$

the second identity by (3.7.8) if $u$ is an energy minimizer and by (3.6.11) if $u$ is an (appropriately rescaled) $F_{\lambda}$-minimizer. This result, together with (3.7.6), implies:

Proposition 3.7.2. If $u$ is either an energy minimizer or an $F_{\lambda}$-minimizer, satisfying (3.6.11), then $L_{+}$has exactly one negative eigenvalue.

Returning to $L_{-}$, we note that

$$
L_{-} u=-\left(\Delta u-\lambda u+|u|^{p-1} u\right)=0,
$$

So

$$
u \in \mathcal{N}\left(L_{-}\right) .
$$

We have the following more precise result.

Proposition 3.7.3. If $u$ is either an energy minimizer or an $F_{\lambda}$-minimizer, satisfying (3.6.11), then

$$
\mathcal{N}\left(L_{-}\right)=\operatorname{Span}(u)
$$

Proof. It suffices to show that any nonzero, real-valued element of $\mathcal{N}\left(L_{-}\right)$must be either everywhere $>0$ or $<0$, since then no two such can be orthogonal to each other. Now, if $v \in \mathcal{N}\left(L_{-}\right)$is real valued and $\|v\|_{L^{2}}=1$, then $v$ minimizes

$$
\left\{\|\nabla v\|_{L^{2}}^{2}+\lambda\|v\|_{L^{2}}^{2}-\left(|u|^{p-1} v, v\right):\|v\|_{L^{2}}=1\right\} .
$$

Then $|v|$ is also minimizing, so $|v| \in \mathcal{N}\left(L_{-}\right)$. Then the Harnack inequality implies $|v|>0$ on $M$, so indeed either $v>0$ or $v<0$ on $M$.

We turn to some comments on $\mathcal{N}\left(L_{+}\right)$. In case $M$ has a 1-parameter group of isometries, generated by a vector field $\mathfrak{X}$ (known as a Killing field), we get an element of $\mathcal{N}\left(L_{+}\right)$as follows. Since Killing fields commute with $\Delta$ we have (assuming $u>0$ )

$$
\Delta(\mathfrak{X} u)-\lambda(\mathfrak{X} u)+p|u|^{p-1}(\mathfrak{X} u)=0,
$$

hence

$$
\mathfrak{X} u \in \mathcal{N}\left(L_{+}\right), \quad \text { if } \mathfrak{X} \text { is a Killing field on } M,
$$

given estimates on $u$ assuring that $\mathfrak{X} u \in \mathcal{D}\left(L_{+}\right)$.

It is useful to regard $L_{+}$and $L_{-}$as two operators in a continuum, defined by

$$
L_{a} \psi=-\Delta \psi+\lambda \psi-a|u|^{p-1} \psi,
$$

for $a \in \mathbb{R}$, particularly for $a \in[1, p]$.. Note that

$$
L_{1}=L_{-}, \quad L_{p}=L_{+} .
$$

For each $a \in \mathbb{R}, L_{a}$ is self-adjoint on $L^{2}(M)$, with domain $\mathcal{D}\left(L_{a}\right)=H^{2}(M)$. The following result extends Proposition 3.7.2,

Proposition 3.7.4. Assume $u>0$ is either an energy minimizer or an $F_{\lambda}$ minimizer, satisfying (3.6.11). Then, if $1<a \leqslant p, L_{a}$ has exactly one negative eigenvalue, and it has multiplicity one. 
Proof. First note that

$$
\begin{aligned}
\left(L_{a} u, u\right) & =\left(L_{1} u+(1-a) u^{p-1} u, u\right) \\
& =-(a-1) \int_{M} u^{p+1} d V
\end{aligned}
$$

which is $<0$ if $a>1$. Next, for $\psi \in H^{1}(M)$,

$$
\begin{aligned}
\left(L_{a} \psi, \psi\right) & =\left(L_{p} \psi+(p-a) u^{p-1} \psi, \psi\right) \\
& =\left(L_{+} \psi, \psi\right)+(p-a) \int_{M} u^{p-1}|\psi|^{2} d V,
\end{aligned}
$$

so, by (3.7.6), if $u$ is an energy minimizer,

$$
(u, \psi)=0 \Longrightarrow\left(L_{a} \psi, \psi\right) \geq 0 \text { if } a \leq p,
$$

while if $u$ is an $F_{\lambda}$-minimizer satisfying (3.6.11),

$$
\left(u^{p}, \psi\right)=0 \Longrightarrow\left(L_{a} \psi, \psi\right) \geq 0 \text { if } a \leq p .
$$

These results prove the proposition.

The result (3.7.7) implies Spec $L_{1} \subset[0, \infty)$, and Proposition 3.7 .4 implies that Ess Spec $L_{a} \subset[0, \infty)$ for $1<a \leq p$. We can say more about the essential spectrum.

Proposition 3.7.5. If $u$ is either an energy minimizer or an $F_{\lambda}$-minimizer satisfying (3.6.11), then, for all $a \in \mathbb{R}$,

$$
\text { Ess Spec } L_{a}=\operatorname{Ess} \operatorname{Spec}(-\Delta+\lambda) \text {. }
$$

Proof. Given $a \in \mathbb{R}$, pick $\mu>0$ so large that $L_{a}+\mu$ and $-\Delta+(\lambda+\mu)$ are both invertible. By Weyl's essential spectrum theorem ([RS78], p. 112) it suffices to note that

$$
S_{a}=\left(L_{a}+\mu\right)^{-1}-(-\Delta+(\lambda+\mu))^{-1} \text { is compact. }
$$

Recalling the formula (3.7.18) for $L_{a}$, we have, by the resolvent identity,

$$
S_{a}=-a\left(L_{a}+\mu\right)^{-1} M_{|u|^{p-1}}(-\Delta+(\lambda+\mu))^{-1},
$$

whose compactness follows readily from the decay results given in 2.6 and 33.4 plus the Rellich theorem.

For the next result, we assume the following:

$$
\operatorname{Spec}(-\Delta+\lambda) \subset[\delta, \infty), \quad \delta>0 .
$$

For $F_{\lambda}$-minimizers, this is equivalent to the hypothesis (1.0.5)-11.0.6). For energy minimizers, (3.7.27) is slightly stronger than (3.7.9), and it can be expected to hold for almost all (if not all) energy minimizers.

Proposition 3.7.6. Let $u$ be either an energy minimizer or an $F_{\lambda}$-minimizer satisfying (3.6.11), and assume (3.7.27) holds. Then

$$
1<a<p \Longrightarrow \mathcal{N}\left(L_{a}\right)=0 \text {. }
$$

Proof. By Proposition 3.7.5 and (3.7.27), for each $a$,

$$
\text { Ess Spec } L_{a} \subset[\delta, \infty), \quad \delta>0 .
$$

Suppose $a_{0} \in(1, p)$ and $\operatorname{dim} \mathcal{N}\left(L_{a_{0}}\right)=m>0$. The Kato-Rellich theorem ([RS78, p. 22) implies there exist analytic functions $\lambda_{j}(a), 1 \leq j \leq m$, for $a$ close to $a_{0}$, 
with $\lambda_{j}\left(a_{0}\right)=0$, such that these are all the eigenvalues of $L_{a}$ near 0 . Also (RS78, p. 71) there are corresponding eigenfunctions $\psi_{j a}$, analytic in $a$ :

$$
L_{a} \psi_{j a}=\lambda_{j}(a) \psi_{j a}, \quad\left(\psi_{j a}, \psi_{k a}\right) \equiv \delta_{j k},
$$

the orthonormality holding for $a$ real (and close to $a_{0}$ ).

Let us denote by $\psi_{a}^{\#}$ the (normalized) eigenfunction of $L_{a}$ given by Proposition 3.7.4. We have

$$
\left(\psi_{j a}, \psi_{a}^{\#}\right)=0,
$$

for $j \in\{1, \ldots, m\}$, real $a$ close to $a_{0}$. Now apply $d / d a$ to (3.7.30). We get

$$
-u^{p-1} \psi_{j a}+L_{a} \xi_{j a}=\lambda_{j}^{\prime}(a) \psi_{j a}+\lambda_{j}(a) \xi_{j a}
$$

where

$$
\xi_{j a}=\frac{d}{d a} \psi_{j a} .
$$

The normalization in (3.7.30) implies

$$
\left(\psi_{j a}, \xi_{j a}\right)=0,
$$

so taking the inner product of (3.7.32) with $\psi_{j a}$ gives

$$
\lambda_{j}^{\prime}(a)\left\|\psi_{j a}\right\|^{2}=-\int_{M} u^{p-1}\left|\psi_{j a}\right|^{2} d V,
$$

since

$$
\left(L_{a} \xi_{j a}, \psi_{j a}\right)=\left(\xi_{j a}, \lambda_{j}(a) \psi_{j a}\right)=0 .
$$

Hence, for $a$ close to 0 ,

$$
\lambda_{j}^{\prime}(a)<0,
$$

and if $\lambda_{j}\left(a_{0}\right)=0$, we get

$$
\lambda_{j}(a)<0 \text { for } a_{0}<a<a_{0}+\epsilon,
$$

for some positive $\epsilon$. This contradicts Proposition 3.7.4 and completes the proof.

3.8. Conditional orbital stability result. We assume $M$ is a weakly homogeneous space, and $p$ satisfies (3.0.1). As in $\$ 3.5$, we fix $\beta>0$ and set

$$
\begin{gathered}
X=\left\{u \in H^{1}(M): Q(u)=\beta\right\}, \quad \mathcal{I}_{\beta}=\inf \{E(u): u \in X\}, \\
Y=\left\{u \in X: E(u)=\mathcal{I}_{\beta}\right\} .
\end{gathered}
$$

Under these hypotheses, the nonlinear Schrödinger equation

$$
i v_{t}+\Delta v+|v|^{p-1} v=0, \quad v(0)=v_{0},
$$

is globally solvable, given $v_{0} \in H^{1}(M)$, via an argument given for $\mathbb{R}^{n}$ in SS99, $\S 3.2 .2$. Conservation of mass and energy imply that $X$ and $Y$ are invariant under the solution operator to (3.8.2). We investigate the following question concerning orbital stability. Assume

$$
v_{0} \in X
$$

is close to $Y$ (distance measured in $H^{1}(M)$-norm). We then ask whether the solution $v(t)$ to (3.8.2) can be shown to be close to $Y$, for all $t \in \mathbb{R}$. Since energy is conserved for solutions to (3.8.2):

$$
E(v(t)) \equiv E\left(v_{0}\right),
$$


a positive result would follow if one could show that if $u \in X$ and $E(u)$ is close to $\mathcal{I}_{\beta}$, then $u$ is close to $Y$.

We establish such a result, under the following two assumptions. The first is an essential uniqueness hypothesis:

$$
\begin{aligned}
& \text { If } u_{1}, u_{2} \text { are positive functions in } Y \text {, there is an isometry } \\
& \varphi: M \rightarrow M \text { such that } u_{2}=u_{1} \circ \varphi \text {. }
\end{aligned}
$$

Recall that if $u \in Y$, there exists $\lambda \in \mathbb{R}$ such that

$$
-\Delta u+\lambda u-|u|^{p-1} u=0 .
$$

The hypothesis (3.8.5) implies that (3.8.6) holds with the same $\lambda$ for all $u \in Y$. Our second hypothesis is that (3.7.27) hold, i.e.,

$$
\operatorname{Spec}(-\Delta+\lambda) \subset[\delta, \infty), \quad \text { for some } \delta>0,
$$

which, recall, is slightly stronger than (3.7.9).

To state our first result, let $\mathcal{G}$ denote the group of operators on functions on $M$ of the form

$$
u(x) \mapsto e^{i \theta} u(\varphi(x)), \quad \theta \in \mathbb{R}, \quad \varphi: M \rightarrow M \text { isometry } .
$$

Thus $\mathcal{G}$ acts as a group of isometries on $L^{2}(M)$ and on $H^{1}(M)$, preserving $X$ and $Y$. The following is immediate.

Proposition 3.8.1. Under the hypothesis (3.8.4), $\mathcal{G}$ acts transitively on $Y$, and $Y$ is a smooth, finite dimensional submanifold of $X$.

It is this result that puts the "orbital" in "orbital stability." In case $M=\mathbb{R}^{n}, Y$ (shown in 4.2 to be nonempty) is diffeomorphic to $\mathbb{R}^{n} \times S^{1}$ (granted hypothesis (3.8.5), also demonstrated for $\mathbb{R}^{n}$ in $\$$ A.2). In other cases, the group of isometries of $M$ might be discrete and $Y$ would be 1-dimensional.

To proceed, for $\epsilon>0$, set

$$
\mathcal{O}_{\epsilon}=\left\{u \in X: \operatorname{dist}_{H^{1}}(u, Y) \leq \epsilon\right\} .
$$

Then $\mathcal{O}_{\epsilon}$ is invariant under the action of $\mathcal{G}$. By Proposition 3.8.1, if $\epsilon$ is sufficiently small, given $u \in Y, \mathcal{O}_{\epsilon}$ is swept out by the $\mathcal{G}$-action on a codimension- $m$ space $\Sigma$, normal to $Y$ at $u$ (with $m=\operatorname{dim} Y$ ).

The following is an orbital stability result.

Proposition 3.8.2. Assume hypotheses (3.8.5) and 3.8.7). For $\epsilon>0$ sufficiently small, the following holds. If $v_{\nu} \in \mathcal{O}_{\epsilon}$ and $E\left(v_{\nu}\right) \rightarrow \mathcal{I}_{\beta}$, then

$$
\operatorname{dist}_{H^{1}}\left(v_{\nu}, Y\right) \rightarrow 0 \text {. }
$$

Note that we can take $\tilde{v}_{\nu} \in \Sigma$ such that $E\left(\tilde{v}_{\nu}\right)=E\left(v_{\nu}\right)$, and $\operatorname{dist}\left(\tilde{v}_{\nu}, Y\right)=$ $\operatorname{dist}\left(v_{\nu}, Y\right)$, so without loss of generality we can assume $v_{\nu} \in \Sigma$. We will parametrize an appropriate space $\Sigma$ by a neighborhood of 0 in an $\mathbb{R}$-linear subspace $V$ of $T_{u} X$, of codimension $m$, as follows. We set

$$
V=\left\{\psi \in T_{u} X: \psi \perp T_{u} Y\right\} .
$$

Recall the characterization of $T_{u} X$ in (3.5.4), supplemented by (3.5.7)-(3.5.8). $\mathrm{V}$ is an $\mathbb{R}$-linear subspace of $H^{1}(M)$, of codimension $m+1$, a Hilbert space with the $H^{1}$-norm. 
To proceed, we define a function $F$ on a neighborhood of $0 \in V$ by

$$
F(\psi)=E\left(a \frac{u+\psi}{\|u+\psi\|}\right) .
$$

We have

$$
F(0)=E(u)=\mathcal{I}_{\beta}, \quad D F(0)=0,
$$

and calculations of $\S 3.5$ give

$$
D^{2} F(0)(\psi, \psi)=\left(L_{+} \psi_{0}, \psi_{0}\right)+\left(L_{-} \psi_{1}, \psi_{1}\right) .
$$

In light of this, Proposition 3.8.2 is a consequence of the following.

Proposition 3.8.3. Let $V$ be a real Hilbert space, $\mathcal{B}_{r}$ a ball of radius $r$ centered at $0 \in V$, and $F: \mathcal{B}_{r} \rightarrow \mathbb{R} a C^{2}$ function satisfying the following conditions:

$$
F(0)=\mathcal{I}_{\beta}, \quad \psi \in \mathcal{B}_{r} \backslash 0 \Rightarrow F(\psi)>\mathcal{I}_{\beta}
$$

(so $D F(0)=0)$. Also assume there exists $C>0$ and an orthogonal projection $P: V \rightarrow V$, with range of finite codimension, such that, for $\psi \in V$,

$$
D^{2} F(0)(\psi, \psi) \geq C\|P \psi\|_{V}^{2} .
$$

Then, if $\rho \in(0, r)$ is small enough,

$$
\psi_{\nu} \in \mathcal{B}_{\rho}, F\left(\psi_{\nu}\right) \rightarrow \mathcal{I}_{\beta} \Longrightarrow\left\|\psi_{\nu}\right\|_{V} \rightarrow 0 .
$$

Proof. Taylor's formula with remainder gives

$$
F(\psi)=\mathcal{I}_{\beta}+\frac{1}{2} D^{2} F(0)(\psi, \psi)+R(\psi),
$$

with

$$
R(\psi)=\int_{0}^{1}\left[D^{2} F(t \psi)-D^{2} F(0)\right](\psi, \psi)(1-t) d t=o\left(\|\psi\|_{V}^{2}\right)
$$

Hence, if $\psi \in \mathcal{B}_{\rho}$ and $\rho$ is small enough,

$$
\begin{aligned}
F(\psi) & \geqslant \mathcal{I}_{\beta}+\frac{C}{2}\|P \psi\|_{V}^{2}-o\left(\|\psi\|_{V}^{2}\right) \\
& \geqslant \mathcal{I}_{\beta}+\frac{C}{4}\|P \psi\|_{V}^{2}-o\left(\left\|P^{\perp} \psi\right\|_{V}^{2}\right),
\end{aligned}
$$

where $P^{\perp}=I-P$ has finite dimensional range. Hence the hypothesis (3.8.17) on $\psi_{\nu}$ implies

$$
\left\|P \psi_{\nu}\right\|_{V} \longrightarrow 0
$$

We need to show that $P^{\perp} \psi_{\nu} \rightarrow 0$ in $W=$ Range $P^{\perp} \subset V$. The sequence $\left(P^{\perp} \psi_{\nu}\right)$ is a bounded sequence in $W$, so $\left(\psi_{\nu}\right)$ has a subsequence (which we continue to denote $\left.\left(\psi_{\nu}\right)\right)$ such that $P^{\perp} \psi_{\nu} \rightarrow \tilde{\psi}$. Hence $\psi_{\nu} \rightarrow \tilde{\psi}$. Now $F\left(\psi_{\nu}\right) \rightarrow \mathcal{I}_{\beta}$ implies $F(\tilde{\psi})=\mathcal{I}_{\beta}$. The hypothesis (3.8.15) then gives $\tilde{\psi}=0$, and completes the proof.

Remark. In the setting of Proposition 3.8.3, the range of $P^{\perp}$ is the orthogonal complement of $T_{u} Y$ in

$$
\left\{\psi=\psi_{0}+i \psi_{1} \in T_{u} X:\left(L_{+} \psi_{0}, \psi_{0}\right)+\left(L_{-} \psi_{1}, \psi_{1}\right)=0\right\},
$$

which is a linear space, by (3.7.6)-(3.7.7), and is finite dimensional, given (3.8.7), by (3.7.29). 
Remark. One setting where Proposition 3.8 .2 applies is that of Euclidean space, $M=\mathbb{R}^{n}$. In this case, the uniqueness hypothesis (3.8.5) and the spectral hypothesis (3.8.7) follow from Proposition A.2.3. In this case, orbital stability was established in Wei86. Further applications of Proposition 3.8.2 are being pursued in CMMT12.

\section{EXPLORATION OF SYMMETRIZATION TECHNIQUES}

As mentioned in the Introduction, works of Str77 and BL83 used a symmetrization technique to construct ground states on Euclidean space, namely $F_{\lambda^{-}}$ minimizers in [Str77] and minimizers of $\|\nabla u\|_{L^{2}}^{2}$ subject to the constraint (1.3.1) in BL83. Here we explore other applications of such a symmetrization technique.

Behind this approach is a key rearrangement lemma. We state this result and say a little about how it has been proved in 4.1 and then proceed to applications in $\S 44.24 .4$

We use the technique to produce $F_{\lambda}$-minimizers on hyperbolic space in 44.2 Here, we make use of arguments from CM10, but with simplifications, which allow us to completely avoid appeal to concentration-compactness arguments. We obtain a unified treatment of $F_{\lambda}$-minimizers on hyperbolic space and on Euclidean space.

In 4.3, we apply the symmetrization technique to the task of maximizing the Weinstein functional. For Euclidean space, this provides a short and direct proof of existence of such maximizers. The dilation structure of Euclidean space plays a crucial role, and we note myriad examples of Riemannian manifolds for which the Weinstein functional does not have a maximum.

In 4.4 we discuss the symmetrization approach to the existence of energy minimizers. In this case, this approach seems to stop short of actually establishing the existence of such minimizers, though we do obtain some interesting information.

4.1. The rearrangement lemma. Here is the key rearrangement lemma.

Lemma 4.1.1. If $M=\mathbb{R}^{n}$ or $\mathcal{H}^{n}$, replacing $u \in H^{1}(M)$ by its radial decreasing rearrangement does not increase $\|\nabla u\|_{L^{2}}$.

Proofs have been given in [Str77] and in [BL83] when $M=\mathbb{R}^{n}$. The result for $M=\mathcal{H}^{n}$ was established in CM10. The proof requires two nontrivial ingredients. One is heat kernel monotonicity. This has been established, on all rank-one symmetric spaces, using exact formulas for the heat kernel.

The other ingredient is an integral rearrangement inequality. This rearrangement inequality holds for $M=\mathbb{R}^{n}$ or $\mathcal{H}^{n}$. In the former case, it is a consequence of a general rearrangement inequality of BLL74, in the latter case, Bec92 produced the extension of such a rearrangement inequality to hyperbolic space. The proof of the rearrangement inequality requires that $M$ be a rank-one symmetric space, and further that $M$ possess reflection symmetry, across a totally geodesic hypersurface. Such reflection symmetry fails for the other noncompact rank-one symmetric spaces.

4.2. Symmetrization approach to $F_{\lambda}$-minimizers. Here we take $M$ to be either $n$-dimensional hyperbolic space $\mathcal{H}^{n}$ or Euclidean space $\mathbb{R}^{n}, n \geqslant 2$. As before, we define $\delta_{0}$ to be the smallest number satisfying

$$
\operatorname{Spec}(-\Delta) \subset\left[\delta_{0}, \infty\right) \text {. }
$$


If $M=\mathbb{R}^{n}$, we have $\delta_{0}=0$. If $M=\mathcal{H}^{n}$, we have $\delta_{0}=(n-1)^{2} / 4$. As in 2 , we assume

$$
\lambda>-\delta_{0}, \quad p+1 \in\left(2, \frac{2 n}{n-2}\right), \quad \beta \in(0, \infty) .
$$

We aim to minimize

$$
F_{\lambda}(u)=\|\nabla u\|_{L^{2}}^{2}+\lambda\|u\|_{L^{2}}^{2},
$$

subject to the constraint

$$
J_{p}(u)=\int_{M}|u|^{p+1} d V=\beta .
$$

This was accomplished in $\$ 2$, in the more general setting of weakly homogeneous spaces. Here we use the symmetrization method.

We turn to the task of finding the desired minimizer. Note that (4.2.1)-44.2.2) imply

$$
F_{\lambda}(u) \approx\|u\|_{H^{1}(M)}^{2},
$$

and in this setting we have the Sobolev embedding result

$$
H^{1}(M) \subset L^{q}(M), \quad \forall q \in\left[2, \frac{2 n}{n-2}\right],
$$

if $n \geq 3, \forall q \in[2, \infty)$ if $n=2$. The results (4.2.5)-(4.2.6) imply

$$
\|u\|_{L^{p+1}}^{2} \leq C F_{\lambda}(u)
$$

So

$$
I_{\beta}=\inf \left\{F_{\lambda}(u): J_{p}(u)=\beta\right\}>0 .
$$

Let $u_{\nu} \in H^{1}(M)$ satisfy

$$
J_{p}\left(u_{\nu}\right)=\beta, \quad F_{\lambda}\left(u_{\nu}\right) \leq I_{\beta}+\frac{1}{\nu} .
$$

Passing to a subsequence, which we continue to denote $\left(u_{\nu}\right)$, we have

$$
u_{\nu} \longrightarrow u \in H^{1}(M), \quad \text { converging weakly. }
$$

Rellich's theorem gives

$$
H^{1}(\Omega) \hookrightarrow L^{p+1}(\Omega) \text { compact, }
$$

for all smoothly bounded $\Omega \subset M$, as long as $p+1$ satisfies (4.2.2), so

$$
u_{\nu} \longrightarrow u, \text { in } L^{p+1}(\Omega) \text { norm, }
$$

for all such $\Omega \subset M$. Fix a base point $o \in M$, and replace $u_{\nu}$ by its radial decreasing rearrangement. By Lemma 4.1.1, this replacement does not increase $\left\|\nabla u_{\nu}\right\|_{L^{2}}$. On the other hand, such a replacement clearly leaves $\left\|u_{\nu}\right\|_{L^{2}}$ fixed, hence lowers $F_{\lambda}\left(u_{\nu}\right)$. It also leaves $J_{p}\left(u_{\nu}\right)$ fixed. Thus we can assume $u_{\nu}(x) \geq 0$, that it is rotationally symmetric about $o \in M$, that it is monotone in $\operatorname{dist}(x, o)$, and that (4.2.9), (4.2.10) and (4.2.12) hold. We need to show that

$$
J_{p}(u)=\beta, \quad \text { i.e., }\|u\|_{L^{p+1}}=\beta^{1 /(p+1)} .
$$

Clearly $J_{p}(u) \leq \beta$ and $F_{\lambda}(u) \leq I_{\beta}$. Given (4.2.13), it would follow from (4.2.8) and (4.2.10) that

$$
F_{\lambda}(u)=I_{\beta}
$$


and also that $H^{1}$-norm convergence holds in (4.2.10).

To demonstrate (4.2.13), let us set $\|u\|_{H^{1}}=\|\nabla u\|_{L^{2}(M)}+\|u\|_{L^{2}(M)}, H_{r}^{1}(M)=$ set of radially symmetric functions in $H^{1}(M), M_{R}=M \backslash B_{R}(o)$, and

$$
J_{R} v=\left.v\right|_{M_{R}} .
$$

We assert the following.

Lemma 4.2.1. Given $q>2$, we have

$$
\lim _{R \rightarrow \infty}\left\|J_{R}\right\|_{\mathcal{L}\left(H_{r}^{1}, L^{q}\right)}=0 .
$$

Given this lemma, we have for the radial sequence $\left(u_{\nu}\right)$ satisfying (4.2.9) that, for each $\epsilon>0$, there exists $R<\infty$ such that

$$
\int_{M_{R}}\left|u_{\nu}\right|^{p+1} d V \leq \epsilon, \quad \forall \nu,
$$

and then (4.2.13) follows from (4.2.12).

It remains to prove Lemmma 4.2.1. If we show that

$$
\lim _{R \rightarrow \infty}\left\|J_{R}\right\|_{\mathcal{L}\left(H_{r}^{1}, L^{\infty}\right)}=0,
$$

then, since for $q>2$

$$
\begin{aligned}
\int_{M_{R}}|v|^{q} d V & \leq\|v\|_{L^{\infty}\left(M_{R}\right)}^{q-2} \int_{M_{R}}|v|^{2} d V \\
& \leq\left\|J_{R} v\right\|_{L^{\infty}}^{q-2}\|v\|_{H^{1}}^{2},
\end{aligned}
$$

we have (4.2.16).

It remains to prove (4.2.18). Here is one approach. We can replace $R$ by $R+1$. Take $\chi_{R} \in \operatorname{Lip}(M), \chi_{R}(x)=0$ for $x \in B_{R}(o), \chi_{R}(x)=\operatorname{dist}\left(x, B_{R}(o)\right)$ for $x \in$ $B_{R+1}(o), \chi_{R}(x)=1$ for $x \in M_{R+1}$. Then, for $v \in H_{r}^{1}(M)$, we have

$$
\chi_{R} v \in H_{0, r}^{1}\left(M_{R}\right)=H_{0}^{1}\left(M_{R}\right) \cap H_{r}^{1}(M),
$$

and

$$
\left\|\nabla\left(\chi_{R} v\right)\right\|_{L^{2}} \leq\|v\|_{H^{1}} .
$$

Hence, in all cases except $M=\mathbb{R}^{2}$, (4.2.18) is a consequence of the following.

Lemma 4.2.2. Except for $M=\mathbb{R}^{2}$, we have

$$
\|v\|_{L^{\infty}} \leq \eta(R)\|\nabla v\|_{L^{2}}, \quad \forall v \in H_{0, r}^{1}\left(M_{R}\right),
$$

with

$$
\lim _{R \rightarrow \infty} \eta(R)=0 .
$$

Proof. Take $v \in H_{0, r}^{1}\left(M_{R}\right)$. Slightly abusing notation, we write $v(x)=v(r)$. Then

$$
\|\nabla v\|_{L^{2}}^{2}=\int_{R}^{\infty}\left|v^{\prime}(r)\right|^{2} A(r) d r
$$

where

(4.2.25) $A(r)=(n-1)$-dimensional area of $\{x \in M: \operatorname{dist}(x, o)=r\}$. 
Now

$$
\begin{aligned}
\|v\|_{L^{\infty}} & \leq \int_{R}^{\infty}\left|v^{\prime}(r)\right| d r \\
& =\int_{r}^{\infty}\left|v^{\prime}(r)\right| A(r)^{1 / 2} A(r)^{-1 / 2} d r \\
& \leq \eta(R)\|\nabla v\|_{L^{2}},
\end{aligned}
$$

by Cauchy's inequality, where

$$
\eta(R)=\left(\int_{R}^{\infty} \frac{d r}{A(r)}\right)^{1 / 2} .
$$

This gives (4.2.22), except when $M=\mathbb{R}^{2}$. In fact, $A(r)=A_{n} r^{n-1}$ when $M=\mathbb{R}^{n}$, and it blows up exponentially when $M$ is $\mathcal{H}^{n}$.

Finally, the case $M=\mathbb{R}^{2}$ of (4.2.18) follows from the next result, given in BL83] as Radial Lemma A.II, which in turn follows Str77.

Lemma 4.2.3. If $M=\mathbb{R}^{n}, n \geq 2$, then, for $R \geq 1$,

$$
\sup _{|x|=R}|v(x)| \leq C_{n} R^{-(n-1) / 2}\|v\|_{H^{1}}, \quad \forall v \in H_{0, r}^{1}\left(M_{1}\right) .
$$

4.3. Symmetrization approach to Weinstein functional maximization. Complementing 4.2 we note how the symmetrization procedure allows for a simplified proof of the existence of a maximum for the Weinstein functional $W(u)$ in (1.3.3), in the Euclidean space setting, $\mathbb{R}^{n}$ (for $n \geqslant 2$ ). Recall,

$$
W(u)=\frac{\|u\|_{L^{p+1}}^{p+1}}{\|u\|_{L^{2}}^{\alpha}\|\nabla u\|_{L^{2}}^{\beta}},
$$

with $\alpha=2-(n-2)(p-1) / 2, \beta=n(p-1) / 2$. We keep the requirement (4.2.2) on $p$. The Gagliardo-Nirenberg estimate implies $W(u)$ is bounded from above. Denote its supremum by $W_{\max }$.

Now, if $u_{\nu} \in H^{1}\left(\mathbb{R}^{n}\right)$ and $W\left(u_{\nu}\right) \rightarrow W_{\max }$, then $W\left(u_{\nu}^{*}\right) \geq W\left(u_{\nu}\right)$ if $u_{\nu}^{*}$ is the radial decreasing rearrangement of $u_{\nu}$, so we need only maximize $W(u)$ over $H_{r}^{1}\left(\mathbb{R}^{n}\right)$. For the next step, we follow the standard argument and use the fact that $W(u)$ is invariant under $u \mapsto a u$ and $u(x) \mapsto u(b x)$ to impose the normalization

$$
\left\|u_{\nu}\right\|_{L^{2}}=1, \quad\left\|\nabla u_{\nu}\right\|_{L^{2}}=1,
$$

so

$$
\left\|u_{\nu}\right\|_{L^{p+1}} \rightarrow W_{\max }^{1 /(p+1)} .
$$

If we pass to a subsequence such that $u_{\nu} \rightarrow u$ weak $^{*}$ in $H^{1}\left(\mathbb{R}^{n}\right)$, results from $\S \mathrm{A} .4$ yield $u_{\nu} \rightarrow u$ in norm in $L^{p+1}\left(\mathbb{R}^{n}\right)$. Also $\|u\|_{L^{2}} \leq 1$ and $\|\nabla u\|_{L^{2}} \leq 1$, so

$$
W(u) \geq W_{\max } .
$$

This requires $W(u)=W_{\max }$ (hence $\|u\|_{L^{2}}=\|\nabla u\|_{L^{2}}=1$, and therefore $u_{\nu} \rightarrow u$ in norm in $H^{1}\left(\mathbb{R}^{n}\right)$.) We have the desired maximizer. A computation of

$$
\left.\frac{d}{d \tau} W(u+\tau v)\right|_{\tau=0}=\frac{(N(u), v)}{\|u\|_{L^{2}}^{2 \alpha}\|\nabla u\|_{L^{2}}^{2 \beta}}
$$

shows that such a maximizer $u$ solves the equation

$$
\Delta u-\lambda u+K u^{p}=0,
$$


with

$$
\lambda=\frac{\alpha}{\beta} \frac{\|\nabla u\|_{L^{2}}^{2}}{\|u\|_{L^{2}}^{2}}, \quad K=\frac{p+1}{\beta} \frac{\|\nabla u\|_{L^{2}}^{2}}{\|u\|_{L^{p+1}}^{p+1}}
$$

hence, with the normalization imposed above,

$$
\lambda=\frac{\alpha}{\beta}, \quad K=\frac{p+1}{\beta W_{\max }} .
$$

By contrast, note the following non-existence result.

Proposition 4.3.1. Let $\Omega \subset \mathbb{R}^{n}$ be a nonempty open set such that $\mathbb{R}^{n} \backslash \Omega$ has positive capacity. Then

$$
\left\{W(u): u \in H_{0}^{1}(\Omega)\right\}
$$

does not achieve a maximum.

Proof. Denote the supremum of (4.3.9) by $W_{\max }^{\Omega}$. Then $W_{\max }^{\Omega} \leq W_{\max }^{\mathbb{R}^{n}}$, since the supremum of (4.3.9) is over a subset of $H^{1}\left(\mathbb{R}^{n}\right)$. On the other hand, taking a maximizer of $W(u)$ over $H^{1}\left(\mathbb{R}^{n}\right)$, dilating it, to be highly concentrated near a point $p \in \Omega$, and using a cutoff, we see that $W_{\max }^{\Omega} \geq W_{\max }^{\mathbb{R}^{n}}$, so in fact $W_{\max }^{\Omega}=W_{\max }^{\mathbb{R}^{n}}$. If $v \in H_{0}^{1}(\Omega)$ and $W(v)=W_{\max }^{\Omega}$, we can replace $v$ by $|v|$ and arrange $v \geq 0$. Then extending $v$ by 0 on $\mathbb{R}^{n} \backslash \Omega$ would yield $u \in H^{1}\left(\mathbb{R}^{n}\right)$ such that $W(u)=W_{\max }^{\mathbb{R}^{n}}$. Then $u \geq 0$ would solve (4.3.6). By Harnack's inequality, that would force $u>0$ on $\mathbb{R}^{n}$, yielding a contradiction.

Turning to the setting of hyperbolic space $\mathcal{H}^{n}$, we do not have dilations, and cannot achieve the normalization (4.3.2), when taking $u_{\nu} \in H^{1}\left(\mathcal{H}^{n}\right)$ such that $W\left(u_{\nu}\right) \rightarrow W_{\max }$. We can arrange that

$$
\left\|\nabla u_{\nu}\right\|_{L^{2}}=1,
$$

which implies $\left\|u_{\nu}\right\|_{L^{2}}$ and $\left\|u_{\nu}\right\|_{L^{p+1}}$ are bounded. Again, $u_{\nu}$ can be arranged to be radial (and decreasing). Take a subsequence $u_{\nu} \rightarrow u$ weak $^{*}$ in $H^{1}(M)$. From here, there are two scenarios to consider. After perhaps passing to a further subsequence, either

$$
\left\|u_{\nu}\right\|_{L^{2}} \longrightarrow A>0, \quad(\text { Case I) }
$$

or

$$
\left\|u_{\nu}\right\|_{L^{2}} \longrightarrow 0, \quad \text { (Case II) }
$$

In Case I, we have

$$
\left\|u_{\nu}\right\|_{L^{p+1}}^{p+1} \longrightarrow A^{\alpha} W_{\max }
$$

and $u_{\nu} \rightarrow u$ in $L^{p+1}$-norm, so $\|u\|_{L^{p+1}}^{p+1}=A^{\alpha} W_{\max }$. Also, $\|u\|_{L^{2}} \leq A$ and $\|\nabla u\|_{L^{2}} \leq$ 1 , so

$$
W(u) \geqslant \frac{A^{\alpha} W_{\max }}{A^{\alpha}}=W_{\max }
$$

Hence

$$
W(u)=W_{\max }, \quad\|u\|_{L^{2}}=A, \quad\|\nabla u\|_{L^{2}}=1,
$$


so $u_{\nu} \rightarrow u$ in $H^{1}$-norm, and we have a Weinstein functional maximizer. It solves the PDE (4.3.6), with $\lambda$ and $K$ given by (4.3.7), i.e., in this case,

$$
\lambda=\frac{\alpha}{\beta} \frac{1}{A^{2}}, \quad K=\frac{p+1}{\beta A^{\alpha} W_{\max }} .
$$

In Case II, we have

$$
\left\|u_{\nu}\right\|_{L^{p+1}} \longrightarrow 0, \quad \text { and } u=0 .
$$

In such a case $\left(u_{\nu}\right)$ does not converge to a $W$-maximizer. Note that, if there is a $W$-maximizer $u$, there must be a sequence $u_{\nu}$ satisfying (4.3.10), $W\left(u_{\nu}\right) \rightarrow W_{\max }$, and (4.3.11) (just take $u_{\nu} \equiv u$ ). Thus we pose the following question. For $M=\mathcal{H}^{n}$, is there a sequence $u_{\nu} \in H_{r}^{1}(M)$ satisfying (4.3.10) and $W\left(u_{\nu}\right) \rightarrow W_{\max }$, such that (4.3.11) holds, or must 4.3.12) hold?

In connection with this, we note that part of the proof of Proposition 4.3.1 extends to give

$$
W_{\max }^{\Omega} \geq W_{\max }^{\mathbb{R}^{n}},
$$

for any Riemannian manifold with boundary $\Omega$, where $W_{\max }^{\Omega}$ is the supremum of (4.3.9). It is tempting to conjecture that

$$
W(u)<W_{\max }^{\mathbb{R}^{n}}, \quad \forall u \in H^{1}\left(\mathcal{H}^{n}\right),
$$

and hence $W_{\max }$ is not achieved in $H^{1}\left(\mathcal{H}^{n}\right)$.

On the other hand, there are Riemannian manifolds with boundary $\Omega$ such that

$$
W_{\max }^{\Omega}>W_{\max }^{\mathbb{R}^{n}} .
$$

One can, for example, let $M$ be a compact, connected Riemannian manifold without boundary and let $\Omega=M \backslash B$, where $B \subset M$ is a small ball. It would be interesting to know whether $W_{\max }^{\Omega}$ can be achieved in such cases.

4.4. Symmetrization approach to energy minimizers. We retain the setting of 4 4.2, and assume $M$ is a (noncompact) $n$-dimensional, rank-one symmetric space with reflection symmetry, i.e., $M=\mathbb{R}^{n}$ or $\mathcal{H}^{n}(n \geqslant 2)$. We require on $p$ the more stringent condition

$$
p \in\left(1,1+\frac{4}{n}\right) .
$$

We fix $\beta>0$, and pick $u_{\nu} \in H^{1}(M)$ such that

$$
Q\left(u_{\nu}\right)=\beta, \quad E\left(u_{\nu}\right) \leqslant \mathcal{I}_{\beta}+\frac{1}{\nu},
$$

with

$$
\begin{aligned}
Q(u) & =\|u\|_{L^{2}}^{2}, \quad E(u)=\frac{1}{2}\|\nabla u\|_{L^{2}}^{2}-\frac{1}{p+1}\|u\|_{L^{p+1}}^{p+1}, \\
\mathcal{I}_{\beta} & =\inf \left\{E(u): u \in H^{1}(M), Q(u)=\beta\right\} .
\end{aligned}
$$

As seen in 93 , this leads to bounds

$$
\left\|u_{\nu}\right\|_{H^{1}},\left\|u_{\nu}\right\|_{L^{p+1}} \leq K<\infty .
$$

To proceed, fix a base point $o \in M$. We make use of Lemma 4.1.1, which implies that, if $M=\mathbb{R}^{n}$ or $\mathcal{H}^{n}$, replacing $u_{\nu}$ by its radial decreasing rearrangement does not increase $\left\|\nabla u_{\nu}\right\|_{L^{2}}$. Also, such a replacement leaves $Q\left(u_{\nu}\right)$ invariant. Thus we can 
assume our minimizing sequence $\left(u_{\nu}\right)$ consists of such radial decreasing functions. Passing to a subsequence, we have

$$
u_{\nu} \longrightarrow u, \text { weak }^{*} \text { in } H^{1}(M),
$$

hence weak ${ }^{*}$ in $L^{2}(M)$ and in $L^{p+1}(M)$. The limit $u$ is radial and decreasing. The next result provides valuable information about $E(u)$.

Proposition 4.4.1. For such a sequence $\left(u_{\nu}\right)$, we have

$$
u_{\nu} \longrightarrow u \text { in } L^{p+1}(M) \text {-norm. }
$$

Proof. As long as $p+1<2 n /(n-2)$ (which is a weaker requirement than (4.4.1)), Rellich's theorem gives

$$
H^{1}\left(B_{R}(o)\right) \hookrightarrow L^{p+1}\left(B_{R}(o)\right) \text { compact, }
$$

for each $R<\infty$, where $B_{R}(o)$ is the ball of radius $R$ centered at $o$. Hence we have

$$
u_{\nu} \longrightarrow u \text { in } L^{p+1}\left(B_{R}(o)\right) \text {-norm, } \quad \forall R<\infty .
$$

To proceed, denote by $H_{r}^{1}(M)$ the space of radially symmetric functions in $H^{1}(M)$. Set

$$
M_{R}=M \backslash B_{R}(o), \quad J_{R} v=\left.v\right|_{M_{R}} .
$$

The following complement to (4.4.8) follows from Lemma 4.2.1 Namely, given $q>2$, we have, for $v \in H_{r}^{1}(M)$,

$$
\left\|J_{R} v\right\|_{L^{q}} \leq \delta_{q}(R)\|v\|_{H_{r}^{1}}, \quad \delta_{q}(R) \rightarrow 0 \text { as } R \rightarrow \infty .
$$

Consequently, we have for the radial sequence $\left(u_{\nu}\right)$ that, for each $\epsilon>0$, there exists $R<\infty$ such that

$$
\int_{M_{R}}\left|u_{\nu}\right|^{p+1} d V \leq \epsilon, \quad \forall \nu,
$$

and then (4.4.6) follows from (4.4.8), proving Proposition 4.4.1.

From (4.4.5) we have $\|\nabla u\|_{L^{2}} \leq \lim \inf \left\|\nabla u_{\nu}\right\|_{L^{2}}$, and this together with (4.4.6) gives

$$
E(u) \leq \liminf _{\nu \rightarrow \infty} E\left(u_{\nu}\right)=\mathcal{I}_{\beta} .
$$

We'd like to know that

$$
Q(u)=\beta .
$$

However, Lemma 4.2.1 requires $q>2$, so it is not clear how to establish (4.4.13) directly.

Consequently, even in the current setting, $M=\mathbb{R}^{n}$ or $\mathcal{H}^{n}$, the energy-minimizer existence result seems to need the concentration-compactness argument given in $\sqrt{3}$ (which at present requires negative energy). 


\section{Appendix A.}

As mentioned in the Introduction, we have four appendices. Appendix A.1 presents the concentration-compactness method of P.-L. Lions, in the setting of a class of measured metric spaces. Appendix A.2 discusses the energy of ground states on Euclidean space. Such solutions are seen to have negative energy, and be energy minimizing, when $1<p<1+4 / n$, but not when $1+4 / n<p<(n+2) /(n-2)$. Appendix A.3 discusses cases when $F_{\lambda}$-minimizers can have positive energy, even for $1<p<1+4 / n$, in noneuclidean settings.

Appendix A.4 exhibits some positive solutions to (1.0.3) that are not $F_{\lambda}$-minimizers, and cases where there are two geometrically inequivalent, positive solutions to this equation.

A.1. The concentration-vanishing-splitting trichotomy of Lions in a general setting. In this section we show the concentration-vanishing-splitting trichotomy of Lions [Lio84a, Lio84b] can be extended in a natural fashion to a metric space setting.

Let $X$ be a metric space and $\left\{\mu_{k}\right\}$ a sequence of Borel probability measures on $X$. For $R \in(0, \infty), y \in X$, set $B_{R}(y)=\{x \in X: d(x, y) \leqslant R\}$ to be the closed ball of radius $R$, centered at $y$. Define

$$
Q_{k}(R)=\sup _{y \in X} \mu_{k}\left(B_{R}(y)\right) .
$$

Of course each $Q_{k}$ is a monotone increasing function of $R$ on $[0, \infty)$, and

$$
\lim _{R \rightarrow \infty} Q_{k}(R)=1
$$

Using a standard diagonalization procedure, we can reduce to a subsequence (which we still denote by $\left.\left\{\mu_{k}\right\}\right)$ such that $Q_{k} \rightarrow Q$ on $\mathbb{Q}^{+}$. The function $Q$ is monotone increasing, so set

$$
\alpha=\lim _{R \rightarrow \infty} Q(R) \in[0,1] .
$$

We examine separately the three cases $\alpha=0, \alpha=1$, and $0<\alpha<1$. We will see that these three cases lead to the phenomena of vanishing, concentration, or splitting respectively. (Observe that Lions labels the third case "dichotomy" rather than splitting.)

Case I: $\alpha=0$. In this case,

$$
\lim _{k \rightarrow \infty} \sup _{y \in X} \mu_{k}\left(B_{R}(y)\right)=0, \quad \forall R<\infty .
$$

This is precisely the case of vanishing.

Case II: $\alpha=1$. In this case, for each $\mu \in(0,1)$, there exists $R=R(\mu)$ such that, for every $k, Q_{k}(R(\mu))>\mu$. That means there exist points $y_{k}(\mu) \in X$ such that

$$
\mu_{k}\left(B_{R(\mu)}\left(y_{k}(\mu)\right)\right)>\mu .
$$

Set $y_{k}=y_{k}(1 / 2)$, and observe that

$$
\mu \geqslant 1 / 2 \Longrightarrow d\left(y_{k}(\mu), y_{k}\right) \leqslant R(1 / 2)+R(\mu) .
$$


This follows by definition, since otherwise there would be two disjoint balls in $M$, each with $\mu_{k}$-measure exceeding $1 / 2$, which contradicts $\mu_{k}$ being a probability measure.

As a consequence, with $y_{k}=y_{k}(1 / 2)$ as above, $\mu \in(1 / 2,1)$, and

$$
\tilde{R}(\mu)=R(1 / 2)+2 R(\mu),
$$

we have for all $k$

$$
\mu_{k}\left(B_{\tilde{R}(\mu)}\left(y_{k}\right)\right)>\mu .
$$

As this holds for each $\mu \in(1 / 2,1)$, this is the phenomenon of concentration.

Case III: $0<\alpha<1$. Pick $\epsilon>0$. Then choose $R \in(0, \infty)$ such that $Q(R)>\alpha-\epsilon$. There exists $k_{0}$ such that for each $k \geqslant k_{0}$,

$$
\alpha-\epsilon<Q_{k}(R)<\alpha+\epsilon \text {. }
$$

We can also choose a sequence $R_{k} \rightarrow \infty$ such that

$$
Q_{k}\left(R_{k}\right) \leqslant \alpha+\epsilon .
$$

By A.1.9), there exist points $y_{k} \in X$ such that

$$
\mu_{k}\left(B_{R}\left(y_{k}\right)\right) \in(\alpha-\epsilon, \alpha+\epsilon) .
$$

Set

$$
E_{k}^{\sharp}=B_{R}\left(y_{k}\right), \quad E_{k}^{b}=X \backslash B_{R_{k}}\left(y_{k}\right) .
$$

Then

$$
\operatorname{dist}\left(E_{k}^{\sharp}, E_{k}^{b}\right) \geqslant R_{k}-R
$$

and

$$
\begin{aligned}
\mu_{k}(X)-\mu_{k}\left(E_{k}^{\sharp}\right)-\mu_{k}\left(E_{k}^{b}\right) & =\mu_{k}\left(B_{R_{k}}\left(y_{k}\right) \backslash B_{R}\left(y_{k}\right)\right) \\
& \leqslant \alpha+\epsilon-(\alpha-\epsilon) \\
& =2 \epsilon .
\end{aligned}
$$

This is the phenomenon of splitting. Observe that (A.1.11) and (A.1.14) imply

$$
\left|\mu_{k}\left(E_{k}^{\sharp}\right)-\alpha\right|<\epsilon, \quad\left|\mu_{k}\left(E_{k}^{b}\right)-(1-\alpha)\right|<3 \epsilon .
$$

A.2. Energy of ground states on Euclidean space. Assume $p \in(1,(n+2) /(n-$ $2)$ ), and let $u_{1}>0$ satisfy

$$
-\Delta u_{1}+u_{1}=u_{1}^{p}, \quad u_{1} \in H^{1}\left(\mathbb{R}^{n}\right)
$$

It follows from [Kwo89] and McL93] that such $u_{1}$ is unique, up to a translation; it is radial and exponentially decreasing at infinity. Such a solution is obtained as an $F_{\lambda}$-minimizer, with $\lambda=1$. If we take $\lambda>0$ and set

$$
u_{\lambda}(x)=\sigma^{2 /(p-1)} u_{1}(\sigma x), \quad \lambda=\sigma^{2},
$$

a calculation gives

$$
-\Delta u_{\lambda}+\lambda u_{\lambda}=u_{\lambda}^{p}
$$

Again, by the results cited above, $u_{\lambda}$ is the unique positive solution in $H^{1}\left(\mathbb{R}^{n}\right)$ to such an equation, up to translation. Calculations give 


$$
\begin{aligned}
\left\|u_{\lambda}\right\|_{L^{2}}^{2} & =\lambda^{2 /(p-1)-n / 2}\left\|u_{1}\right\|_{L^{2}}^{2}, \\
\left\|\nabla u_{\lambda}\right\|_{L^{2}}^{2} & =\lambda^{1+2 /(p-1)-n / 2}\left\|\nabla u_{1}\right\|_{L^{2}}^{2}, \\
\int\left|u_{\lambda}\right|^{p+1} d x & =\lambda^{1+2 /(p-1)-n / 2} \int\left|u_{1}\right|^{p+1} d x,
\end{aligned}
$$

hence

$$
E\left(u_{\lambda}\right)=\lambda^{1+2 /(p-1)-n / 2} E\left(u_{1}\right),
$$

while

$$
Q\left(u_{\lambda}\right)=\lambda^{2 /(p-1)-n / 2} Q\left(u_{1}\right) .
$$

Note that

$$
\begin{array}{r}
1+\frac{2}{p-1}-\frac{n}{2}>0 \Longleftrightarrow p<\frac{n+2}{n-2}, \\
\frac{2}{p-1}-\frac{n}{2}>0 \Longleftrightarrow p<1+\frac{4}{n} .
\end{array}
$$

Given $p>1$, the second restriction on $p$ in (A.2.7) is equivalent to (1.0.12). If we set

$$
e(\lambda)=E\left(u_{\lambda}\right), \quad q(\lambda)=\frac{1}{2}\left\|u_{\lambda}\right\|_{L^{2}}^{2},
$$

we get

$$
e(\lambda)=\lambda^{1+\gamma} e(1), \quad q(\lambda)=\lambda^{\gamma} q(1), \quad \gamma=\frac{2}{p-1}-\frac{n}{2} .
$$

The following general result can be combined with A.2.9 to provide further information on $e(\lambda)$ and $q(\lambda)$. (Further consequences are discussed in [CMMT12.)

Proposition A.2.1. Let $M$ be a complete Riemannian manifold and $u_{\lambda}$ a smooth family of positive functions in $H^{1}(M)$, satisfying (A.2.3). Define $q(\lambda)$ and $e(\lambda)$ by (A.2.8). Then

$$
\frac{d e}{d \lambda}=-\lambda \frac{d q}{d \lambda} .
$$

Proof. Take the inner product of A.2.3 with $\partial_{\lambda} u_{\lambda}$, to get

$$
\left(\Delta u_{\lambda}-\lambda u_{\lambda}+u_{\lambda}^{p}, \partial_{\lambda} u_{\lambda}\right)=0 .
$$

Note that

$$
\frac{d q}{d \lambda}=\frac{1}{2} \frac{\partial}{\partial \lambda}\left\|u_{\lambda}\right\|_{L^{2}}^{2}=\left(u_{\lambda}, \partial_{\lambda} u_{\lambda}\right)
$$

Also,

$$
\begin{aligned}
\frac{1}{2} \frac{\partial}{\partial \lambda}\left\|\nabla u_{\lambda}\right\|_{L^{2}}^{2} & =\left(\nabla u_{\lambda}, \nabla \partial_{\lambda} u_{\lambda}\right) \\
& =-\left(\Delta u_{\lambda}, \partial_{\lambda} u_{\lambda}\right)
\end{aligned}
$$

and

$$
\frac{1}{p+1} \frac{\partial}{\partial \lambda} \int_{M} u_{\lambda}^{p+1} d V=\int_{M} u_{\lambda}^{p} \partial_{\lambda} u_{\lambda} d V
$$


SO

$$
\frac{d e_{\lambda}}{d \lambda}=-\left(\Delta u_{\lambda}+u_{\lambda}^{p}, \partial_{\lambda} u_{\lambda}\right) .
$$

Comparing (A.2.12) and (A.2.15) gives (A.2.10), via (A.2.11).

From this, we can deduce information about the sign of $e(1)$, hence of $e(\lambda)$ for all $\lambda>0$. By (A.2.9) and (A.2.7),

$$
\begin{aligned}
1<p<1+\frac{4}{n} & \Longrightarrow q^{\prime}(\lambda)>0, \quad \forall \lambda>0 \\
& \Longrightarrow e^{\prime}(\lambda)<0 \\
& \Longrightarrow e(1)<0 \\
& \Longrightarrow E\left(u_{\lambda}\right)<0, \quad \forall \lambda>0 .
\end{aligned}
$$

On the other hand,

$$
\begin{aligned}
1+\frac{4}{n}<p<\frac{n+2}{n-2} & \Longrightarrow q^{\prime}(\lambda)<0, \quad \forall \lambda>0 \\
& \Longrightarrow e^{\prime}(\lambda)>0 \\
& \Longrightarrow e(1)>0 \\
& \Longrightarrow E\left(u_{\lambda}\right)>0, \quad \forall \lambda>0 .
\end{aligned}
$$

(In the setting of (A.2.17), (1.0.12) is violated, and results of 93 do not apply.) From these observations, we can obtain positive and negative results about energy minimizers. Here is a positive result.

Proposition A.2.2. If $p$ satisfies (A.2.16) and $\lambda>0$, then the positive solution $u \in H^{1}\left(\mathbb{R}^{n}\right)$ to (A.2.3) is energy minimizing, within its mass class.

Proof. Say $\left\|u_{\lambda}\right\|_{L^{2}}^{2}=\beta(\lambda)=2 q(\lambda)$. Results of $\$ 3$ imply there exists a minimizer $v_{\lambda}$ for $E(v)$, subject to the constraint $\|v\|_{L^{2}}^{2}=\beta(\lambda)$. We can assume $v_{\lambda}>0$. Furthermore, there exists $\mu \in \mathbb{R}$ such that

$$
-\Delta v_{\lambda}+\mu v_{\lambda}=v_{\lambda}^{p} \text {. }
$$

We know that $\operatorname{Spec}(-\Delta+\mu) \subset[0, \infty)$, so, in this setting, $\mu \geqslant 0$. There are two possibilities: $\mu>0$ or $\mu=0$. If $\mu>0$, the uniqueness result of Kwo89, McL93. implies $v_{\lambda}=u_{\mu}$, up to a translation. Now $\left\|v_{\lambda}\right\|_{L^{2}}^{2}=\left\|u_{\mu}\right\|_{L^{2}}^{2}=\left\|u_{\lambda}\right\|_{L^{2}}^{2}$ implies $\mu=\lambda$, by (A.2.4). To finish, we claim that $\mu=0$ is impossible. Indeed, if this held, we could take the inner product of (A.2.18) with $v_{\lambda}$ to get

$$
\left\|\nabla v_{\lambda}\right\|_{L^{2}}^{2}=\left\|v_{\lambda}\right\|_{L^{p+1}}^{p+1}
$$

hence

$$
\begin{aligned}
E\left(v_{\lambda}\right) & =\frac{1}{2}\left\|\nabla v_{\lambda}\right\|_{L^{2}}^{2}-\frac{1}{p+1}\left\|v_{\lambda}\right\|_{L^{p+1}}^{p+1} \\
& =\left(\frac{1}{2}-\frac{1}{p+1}\right)\left\|v_{\lambda}\right\|_{L^{p+1}}^{p+1} \\
& >0 .
\end{aligned}
$$

However, the minimum energy in this situation is $<0$, by (A.2.16), so the energy minimizer cannot satisfy (A.2.18) with $\mu=0$.

From this, we have the following existence and uniqueness result. 
Proposition A.2.3. If $p$ satisfies A.2.16) and $\beta>0$, there is a positive $u \in$ $H^{1}\left(\mathbb{R}^{n}\right)$ that minimizes $E(u)$ subject to the constraint $\|u\|_{L^{2}}^{2}=\beta$. This function solves (A.2.3), for some $\lambda>0$, uniquely determined by $\beta$, and it is unique up to translations.

Proof. As seen in (A.2.20), we cannot have $\lambda=0$. It only remains to remark that (A.2.9) sets up the unique correspondence between $\beta$ and $\lambda$.

We now record some negative results, when $p$ satisfies (A.2.17). Here is a preliminary result, using the fact (cf. (3.0.11)) that

$$
\mathcal{I}_{\beta} \rightarrow-\infty \text { as } \beta \rightarrow+\infty \text {. }
$$

Lemma A.2.4. If $p$ satisfies (A.2.17), then, as $\lambda \searrow 0, u_{\lambda}$ is not energy minimizing within its mass class.

Proof. If (A.2.17) holds, as $\lambda \searrow 0,\left\|u_{\lambda}\right\|_{L^{2}} \nearrow+\infty$, so

$$
\inf \left\{E(u):\|u\|_{L^{2}}=\left\|u_{\lambda}\right\|_{L^{2}}\right\} \rightarrow-\infty,
$$

by (A.2.21). Thus, by (A.2.17), $E\left(u_{\lambda}\right)$ is not minimal.

We can extend this result, as follows. Given $\varphi_{1} \in H^{1}\left(\mathbb{R}^{n}\right)$, set

$$
\varphi_{\lambda}(x)=\sigma^{2 /(p-1)} \varphi_{1}(\sigma x), \quad \lambda=\sigma^{2},
$$

for $\lambda \in(0, \infty)$. As in A.2.4 A.2.6),

$$
\left\|\varphi_{\lambda}\right\|_{L^{2}}^{2}=\lambda^{\gamma}\left\|\varphi_{1}\right\|_{L^{2}}^{2}, \quad E\left(\varphi_{\lambda}\right)=\lambda^{1+\gamma} E\left(\varphi_{1}\right)
$$

with $\gamma$ given in A.2.9). Given $\mu>0$ small enough, pick $\varphi_{1} \in H^{1}\left(\mathbb{R}^{n}\right)$ such that (with $\varphi_{\mu}$ as in (A.2.24) and $u_{\mu}$ as in A.2.2), where $\mu$ replaces $\lambda$ )

$$
\left\|\varphi_{\mu}\right\|_{L^{2}}=\left\|u_{\mu}\right\|_{L^{2}}, \quad E\left(\varphi_{\mu}\right)<0
$$

Then

$$
\left\|\varphi_{\lambda}\right\|_{L^{2}}=\left\|u_{\lambda}\right\|_{L^{2}}, \quad E\left(\varphi_{\lambda}\right)<0, \quad \forall \lambda \in(0, \infty) .
$$

This shows that, even for $p$ as in A.2.17,

$$
\mathcal{I}_{\beta}<0, \quad \forall \beta>0 .
$$

Hence, for each $\lambda>0$, the positive solution $u_{\lambda} \in H^{1}\left(\mathbb{R}^{n}\right)$ to A.2.3 is not energy minimizing within its mass class. Here is a stronger result.

Proposition A.2.5. If $p$ satisfies (A.2.17), there is no energy minimizer $u \in$ $H^{1}\left(\mathbb{R}^{n}\right)$ within its mass class, at any positive mass.

Proof. Without loss of generality, such a minimizer can be taken to be $\geqslant 0$. By A.2.27, such a minimizer must have negative energy. It also must solve A.2.3 for some $\lambda \geqslant 0$. If $\lambda>0$, we contradict the conclusion of (A.2.17), and if $\lambda=0$ we contradict (A.2.20). 
A.3. Ground states with positive energy. If $M$ is a weakly homogeneous space of dimension $n$, and

$$
\operatorname{Spec}(-\Delta) \subset\left[\delta_{0}, \infty\right), \quad \lambda>-\delta_{0}, \quad 1<p<\frac{n+2}{n-2},
$$

then, as shown in $₫ 2$, one can minimize $F_{\lambda}(u)=\|\nabla u\|_{L^{2}}^{2}+\lambda\|u\|_{L^{2}}^{2}$, subject to $\|u\|_{L^{p+1}}$ being fixed, and multiply by a constant to get a positive solution to

$$
\Delta u_{\lambda}-\lambda u_{\lambda}+\left|u_{\lambda}\right|^{p-1} u_{\lambda}=0 .
$$

As seen in 4 , when $M=\mathbb{R}^{n}$ (where $\delta_{0}=0$ ), these ground states all have negative energy when $1<p<1+4 / n$, and positive energy when $1+4 / n<p<(n+2) /(n-2)$. Here we note that some of these "ground state" solutions can have positive energy, whenever A.3.1 holds with $\delta_{0}>0$, even when $1<p<1+4 / n$.

In fact, A.3.2 implies

$$
\left\|\nabla u_{\lambda}\right\|_{L^{2}}^{2}=-\lambda\left\|u_{\lambda}\right\|_{L^{2}}^{2}+\int_{M}\left|u_{\lambda}\right|^{p+1} d V
$$

which in turn implies

$$
\begin{aligned}
E\left(u_{\lambda}\right) & =\frac{1}{2}\left\|\nabla u_{\lambda}\right\|_{L^{2}}^{2}-\frac{1}{p+1} \int_{M}\left|u_{\lambda}\right|^{p+1} d V \\
& =-\frac{\lambda}{2}\left\|u_{\lambda}\right\|_{L^{2}}^{2}+\frac{p-1}{2(p+1)} \int_{M}\left|u_{\lambda}\right|^{p+1} d V .
\end{aligned}
$$

If A.3.1 holds with $\delta_{0}>0$, we can pick $\lambda \in\left(-\delta_{0}, 0\right]$ and find a ground state solution to (A.3.2) (i.e., an $F_{\lambda}$-minimizer), and then (A.3.4) gives

$$
\lambda \leq 0 \Longrightarrow E\left(u_{\lambda}\right)>0 .
$$

For example, all the ground states on hyperbolic space $\mathcal{H}^{n}$ associated to $\lambda \leq 0$ have positive energy. It would be interesting to investigate when they are energy minimizing, within their mass class.

A.4. Non- $F_{\lambda}$-minimizers and related non-uniqueness. Fix $\lambda>0, \beta>0, n \geq$ $2, p \in(1,(n+2) /(n-2))$, and $R>0$, and set

$$
\begin{aligned}
& M=\left\{x \in \mathbb{R}^{n}:|x| \geqslant R\right\}, \\
& I_{\beta}=\inf \left\{F_{\lambda}(u): u \in H_{0}^{1}(M), J_{p}(u)=\beta\right\} .
\end{aligned}
$$

As seen in 2.3 , there is no minimizer in such a case. In fact, $I_{\beta}$, given by (A.4.1), is equal to

$$
\inf \left\{F_{\lambda}(u): u \in H^{1}\left(\mathbb{R}^{n}\right), J_{p}(u)=\beta\right\},
$$

and $F_{\lambda}(u)>I_{\beta}$ for all $u \in H_{0}^{1}(M)$. On the other hand, methods of 44.2 readily work to produce a minimizer for $F_{\lambda}$ restricted to the space $H_{0, r}^{1}(M)$ of radial functions in $H_{0}^{1}(M)$, thus achieving

$$
\begin{aligned}
& F_{\lambda}(v)=R_{\beta}, \quad v \in H_{0, r}^{1}(M), \\
& R_{\beta}=\inf \left\{F_{\lambda}(u): u \in H_{0, r}^{1}(M), J_{p}(u)=\beta\right\} .
\end{aligned}
$$

We can arrange that $v \geq 0$ on $M$. Then $v$ is a radial solution to

$$
-\Delta v+\lambda v=K v^{p}, \quad K=\beta^{-1} R_{\beta},\left.\quad v\right|_{\partial M}=0,
$$


and $v>0$ on the interior of $M$. (Cf. (1.1.4)-1.1.5) for the computation of $K$.) Of course, $v$ is not an $F_{\lambda}$-minimizer:

$$
F_{\lambda}(v)>I_{\beta}
$$

We next construct some solutions on a compact, annular region. With $\lambda, \beta, n, p$, and $R$ as above, pick $S>R$ so large that one can take a Euclidean $F_{\lambda}$-minimizer, translate it to be concentrated near a point $p$, satisfying $|p| \sim(R+S) / 2$, and cut it off near $|x|=R$ and $|x|=S$, in such a way as to obtain

$$
\tilde{u} \in H_{0}^{1}(\Omega), \quad J_{p}(\tilde{u})=\beta, \quad F_{\lambda}(\tilde{u})<R_{\beta},
$$

where

$$
\Omega=\left\{x \in \mathbb{R}^{n}: R \leqslant|x| \leqslant S\right\} .
$$

Now, since $\Omega$ is compact, we can find minimizers for each of the following:

$$
\begin{aligned}
& \left.L_{1}=\inf \left\{F_{\lambda}(u): u \in H_{0}^{1}(\Omega), J_{p}(u)=\beta\right)\right\}, \\
& L_{2}=\inf \left\{F_{\lambda}(u): u \in H_{0, r}^{1}(\Omega), J_{p}(u)=\beta\right\} .
\end{aligned}
$$

Note that

$$
L_{1}<R_{\beta}<L_{2}
$$

We can find

$$
\begin{array}{rll}
w_{1} \in H_{0}^{1}(\Omega), & J_{p}\left(w_{1}\right)=\beta, & F_{\lambda}\left(w_{1}\right)=L_{1}, \\
w_{2} \in H_{0, r}^{1}(\Omega), & J_{p}\left(w_{2}\right)=\beta, & F_{\lambda}\left(w_{2}\right)=L_{2},
\end{array}
$$

and arrange that $w_{j} \geq 0$ on $\Omega$. Then $w_{j}$ are positive solutions on $\Omega$ to

$$
-\Delta w_{j}+\lambda w_{j}=K_{j} w_{j}^{p}, \quad K_{j}=\beta^{-1} L_{j},\left.\quad w_{j}\right|_{\partial \Omega}=0 .
$$

Then

$$
u_{j}=K_{j}^{1 /(p-1)} w_{j} \in H_{0}^{1}(\Omega)
$$

are positive solutions to

$$
-\Delta u_{j}+\lambda u_{j}=u_{j}^{p},\left.\quad u_{j}\right|_{\partial \Omega}=0,
$$

and, since A.4.8) implies $K_{1}<K_{2}$, while $J_{p}\left(w_{1}\right)=J_{p}\left(w_{2}\right)$,

$$
\left\|u_{1}\right\|_{L^{p+1}}<\left\|u_{2}\right\|_{L^{p+1}},
$$

so these solutions are geometrically distinct.

Remark. If $\lambda$ is large, the Euclidean $F_{\lambda}$-minimizer is highly peaked, and $S$ need not be much larger than $R$.

\section{REFERENCES}

[Bec92] W. Beckner. Sobolev inequalities, the poisson semigroup, and analysis on the sphere $S^{n}$. Proc. Nat. Acad. Sci. USA, 89:4816-4819, 1992.

[BL83] H. Berestycki and P.-L. Lions. Nonlinear scalar field equations, I, existence of a ground state. Arch. Rat. Mech. Anal., 82:313-345, 1983.

[BLL74] H. Brascamp, E. Lieb, and J. Luttinger. A general rearrangement inequality for multiple integrals. J. Funct. Anal., 17:227-237, 1974.

[BT76] A. Baernstein and B. Taylor. Spherical rearrngements, subharmonic functions, and *-functions in $n$-space. Duke Math. J., 43:245-268, 1976.

[CJ93] C. Clemens and C.K.R.T. Jones. A geometric proof of the Kwong-McLeod uniqueness result. SIAM J. Math. Anal., 24:436-443, 1993. 
[CL82] T. Cazenave and P.-L. Lions. Orbital stability of standing waves for some nonlinear Schrödinger equations. Comm. Math. Phys., 85(4):549-561, 1982.

[CM10] Hans Christianson and Jeremy L. Marzuola. Existence and stability of solitons for the nonlinear Schrödinger equation on hyperbolic space. Nonlinearity, 23(1):89-106, 2010 .

[CMMT12] H. Christianson, J. Marzuola, J. Metcalfe, and M. Taylor. Stability of energyminimizing standing waves, in preparation. 2012.

[GSS90] M. Grillakis, J. Shatah, and W. Strauss. Stability theory of solitary waves in the presence of symmetry II. J. Funct. Anal., 94:308-348, 1990.

[GT01] David Gilbarg and Neil S. Trudinger. Elliptic partial differential equations of second order. Classics in Mathematics. Springer-Verlag, Berlin, 2001. Reprint of the 1998 edition.

[Kwo89] M. Kwong. Uniqueness of positive solutions of $\Delta u-u+u^{p}=0$ in $R^{n}$. Arch. Rat. Mech. Anal., 105:243-266, 1989.

[Lio84a] P.-L. Lions. The concentration-compactness principle in the calculus of variations. The locally compact case. I. Ann. Inst. H. Poincaré Anal. Non Linéaire, 1(2):109-145, 1984.

[Lio84b] P.-L. Lions. The concentration-compactness principle in the calculus of variations. The locally compact case. II. Ann. Inst. H. Poincaré Anal. Non Linéaire, 1(4):223-283, 1984.

[McL93] Kevin McLeod. Uniqueness of positive radial solutions of $\Delta u+f(u)=0$ in $\mathbf{R}^{n}$. II. Trans. Amer. Math. Soc., 339(2):495-505, 1993.

[MS08] G. Mancini and K. Sandeep. On a semilinear equation in $H^{n}$. Ann. Scuola Norm. Sup. Pisa, 7:635-671, 2008.

[Poh65] S. Pohozaev. Eigenfunctions of the equation $\Delta u+\lambda f(u)=0$. Soviet Math., 5:14081411, 1965.

[RS78] M. Reed and B. Simon. Methods of mathematical physics, vol. 4. Academic Press Inc., New York, 1978.

[Sch09] W. Schlag. Stable manifolds for an orbitally unstable NLS. Annals of Math, 169:139227, 2009.

[SS99] Catherine Sulem and Pierre-Louis Sulem. The nonlinear Schrödinger equation, volume 139 of Applied Mathematical Sciences. Springer-Verlag, New York, 1999. Selffocusing and wave collapse.

[Str77] W. Strauss. Existence of solitary waves in higher dimensions. Comm. Math. Phys., 55:149-162, 1977.

[Tao06] Terence Tao. Nonlinear dispersive equations, volume 106 of CBMS Regional Conference Series in Mathematics. Published for the Conference Board of the Mathematical Sciences, Washington, DC, 2006. Local and global analysis.

[Tay11] M. Taylor. Regularity of standing waves on Lipschitz domains. Preprint, 2011.

[Wei83] M. Weinstein. Nonlinear Schrödinger equations and sharp interpolation estimates. Commun. Math. Phys., 87:567-576, 1983.

[Wei85] M. Weinstein. Modulational stability of ground states of nonlinear Schrödinger equations. SIAM J. Math. Anal., 16:472-491, 1985.

[Wei86] M. Weinstein. Lyapunov stability of ground states of nonlinear dispersive evolution equations. Comm. Pure Appl. Math., 39:51-68, 1986.

E-mail address: hans@math.unc.edu

Department of Mathematics, UnC-Chapel Hill, CB\#3250 Phillips Hall, Chapel Hill, NC 27599

E-mail address: marzuola@math.unc.edu

Department of Mathematics, UnC-Chapel Hill, CB\#3250 Phillips Hall, Chapel Hill, NC 27599

E-mail address: metcalfe@email.unc.edu 
Department of Mathematics, UnC-Chapel Hill, CB\#3250 Phillips Hall, Chapel Hill, NC 27599

E-mail address: met@math.unc.edu

Department of Mathematics, UnC-Chapel Hill, CB\#3250 Phillips Hall, Chapel Hill, NC 27599 